\title{
On some one parameter families of genus 2 algebraic curves and half twists
}

\author{
Robert Silhol
}

\begin{abstract}
In this paper we show that for certain families of surfaces in genus 2 there is an action of $\mathrm{PSL}_{2}(\mathbb{Z})$ that can be expressed very naturally both in terms of Fenchel-Nielsen coordinates for the surfaces and in terms of equations of the associated algebraic curves. We also show that one of these families coincides with the $\mathrm{SL}_{2}(\mathbb{R})$ orbit of the translation surface tiled by three squares and that the above $\mathrm{PSL}_{2}(\mathbb{Z})$ action is exactly induced by the natural action of $\mathrm{SL}_{2}(\mathbb{Z})$ on this orbit.
\end{abstract}

Mathematics Subject Classification (2000). 30F10, 14H15, 32G15, 30F30.

Keywords. Hyperbolic surfaces, algebraic curves, translation surfaces, Teichmüller spaces, uniformization.

\section{Introduction}

In recent years one parameter families of algebraic curves in genus 2 have attracted great interest (see for example [Mc] and the bibliography quoted there). Although the families we consider here also define Teichmüller disks the point of view we will take in this paper is somewhat different. We will be interested in families for which one can both explicitly describe the hyperbolic structure and give the form of the equations. The hyperbolic structure will be described by Fenchel-Nielsen coordinates depending in each case on two real parameters, the length of a specific geodesic and a twist parameter. The equations will depend on one complex parameter. Two such families are well known, surfaces with an order 3 automorphism and surfaces with an order 4 automorphism, we will consider these but we will also consider others, two in detail, chosen for the simplicity of both equation and Fenchel-Nielsen coordinates, and give indications on some more.

The important point and one of the main aspect of this paper is that all these families share the fact that there is a natural action of $\operatorname{PSL}_{2}(\mathbb{Z})$ on the Teichmüller subspace, defined by the set of Fenchel-Nielsen coordinates, that yields an explicitly describable action of the permutation group $\mathfrak{S}_{3}$ on the parameter space for the equations. 
To be more specific let

$$
T=\left(\begin{array}{ll}
1 & 0 \\
1 & 1
\end{array}\right) \quad \text { and } \quad R=\left(\begin{array}{cc}
0 & -1 \\
1 & 0
\end{array}\right)
$$

be representatives in $\mathrm{SL}_{2}(\mathbb{Z})$ of the standard generators of $\mathrm{PSL}_{2}(\mathbb{Z})$. Then in all cases $T$ will act by half-Dehn twists along certain geodesics. The action of $R$ is more difficult to describe in full generality but is always based on variants of the following. In [Bu-Si2] it was shown how to associate to Fenchel-Nielsen coordinates a well defined hyperbolic octagon such that the surface is obtained by identifications of opposite edges of the octagon (see Section 1 for details). Conversely from such an octagon we can recover Fenchel-Nielsen coordinates. In the simplest cases $R$ corresponds to a rotation of the octagon performing a circular permutation on 4 of the Weierstrass points.

In all cases the induced action of $T$ on the corresponding subspace of moduli space is generically non-trivial. In the simple cases, as above, the induced action of $R$ will be trivial on moduli space but not on a double cover that will serve as parameter space for the equations. On the other hand $T R$ will again act by half-Dehn twists along another set of geodesics and the induced action will be generically non-trivial on moduli space and distinct from that of $T$. In the not so simple cases where the action of $R$ is more intricate it will turn out that the induced action of $R$ itself is sometimes non-trivial on moduli space (see Sections 3 and 6).

Returning to the point of view of $[\mathrm{Mc}]$ we note that there are also families of translation surfaces with a natural action of $\mathrm{SL}_{2}(\mathbb{Z})$, precisely those described by a theorem of Gutkin and Judge [Gu-Ju] and obtained as $\mathrm{SL}_{2}(\mathbb{R})$ orbits of square-tiled surfaces also called origami (see for example [Hu-Le1]). In Section 5 we will show that one of the families we have constructed coincides precisely with the family of translation surfaces obtained from three squares giving for this family both equations and a description of the hyperbolic geometry. Since this is probably the most striking result of this paper we briefly summarize it here. Consider the L shaped polygon obtained by pasting three Euclidean squares (see Figure 10). Identify the sides of the polygon using horizontal or vertical translations. We obtain in this way a Riemann surface. Moreover since $d z$ is translation invariant the surface comes equipped with a holomorphic differential. Replacing the squares by their images under $\mathrm{SL}_{2}(\mathbb{R})$ we obtain from this construction a one complex parameter family of surfaces. Our results are then,

Theorem A. Let $(S, \omega)$ be in the $\mathrm{SL}_{2}(\mathbb{R})$ orbit of the L shape translation surface tiled by three squares. Then the surface $S$ has Fenchel-Nielsen coordinates of the form

$$
\left(2 \ell, t w, \ell, 2 t w, \ell^{\prime \prime}, 0\right) \text { and } \cosh \left(\ell^{\prime \prime} / 2\right)=2 \cosh (\ell / 2)
$$


and the surface $S$ has an equation of the form

$$
y^{2}=x(x-1)\left(x^{3}+a x^{2}-\frac{8}{3} a x+\frac{16}{9} a\right) \quad \text { with } \omega=\lambda \frac{x d x}{y} .
$$

Theorem B. If $(S, \omega), \ell$, tw and $\ell^{\prime \prime}$ are as in Theorem $A$, then the transform $\left(S^{\prime}, \omega^{\prime}\right)$ of $(S, \omega)$ under $\left(\begin{array}{ll}1 & 0 \\ 1 & 1\end{array}\right)$, has Fenchel-Nielsen coordinates

$$
\left(2 \ell, t w-\frac{1}{2}, \ell, 2 t w-1, \ell^{\prime \prime}, 0\right)
$$

and the transform $\left(S^{\prime \prime}, \omega^{\prime \prime}\right)$ of $(S, \omega)$ under $\left(\begin{array}{cc}0 & -1 \\ 1 & 0\end{array}\right)$ has Fenchel-Nielsen coordinates

$$
\left(2 \ell_{2}, t w_{2}, \ell_{2}, 2 t w_{2}, \ell_{2}^{\prime \prime}, 0\right)
$$

where $\cosh \left(\ell_{2}^{\prime \prime} / 2\right)=2 \cosh \left(\ell_{2} / 2\right)$ and $($ with $L=\cosh (\ell)$ and $T w=\cosh (t w \cdot \ell))$

$$
\begin{aligned}
\cosh \left(\ell_{2}\right)=L_{2} & =T w^{2} \frac{2 L+1}{L-1}-1, \\
T w_{2} & =\sqrt{\frac{2 T w^{2} L^{2}+3 T w^{2} L+T w^{2}-2 L^{2}+2}{4 T w^{2} L+2 T w^{2}-L+1}}, \\
t w_{2} & =-\operatorname{sign}(t w) \operatorname{arccosh}\left(T w_{2}\right) / \operatorname{arccosh}\left(L_{2}\right) .
\end{aligned}
$$

The methods used in this paper are largely inspired by the generalization found by Aline Aigon [Ai] for the $D_{5}$ action on the two parameter space of genus 2 curves with a non-hyperelliptic involution described in [Bu-Si1].

Finally I would like to thank $\mathrm{H}$. Akrout and S. Lelièvre for very useful discussions. Others have also helped in various ways and I would also like to thank A. Aigon, P. Buser, M. Herzlich, C. Mercat and P.-L. Montagard.

\section{Octagons, equations and the basic half-twist transformations}

We proceed to describe in this section the basic tools that we will use throughout this paper.

There are many ways to define genus 2 hyperbolic surfaces, but in the sequel we will describe then in terms of fundamental polygons, an octagon in Sections 2 and 3 a dodecagon in Section 4. On the other hand a more synthetic and practical way to describe a surface is Fenchel-Nielsen coordinates. None of these descriptions are unique but we will need to associate to a given set of Fenchel-Nielsen coordinates an explicit and uniquely defined octagon or dodecagon. For octagons we will do this in two ways (in fact those given in [Bu-Si2]) one (the most used) that we proceed to 
describe here leaving the second to Section 3 and the representations by dodecagons to Section 4.

For this we first note that to a genus 2 hyperbolic surface we can always associate a hyperbolic octagon in the unit disk, symmetric with respect to the origin and such that the sum of the interior angles is $2 \pi$. The corresponding fuchsian group is the group generated by identification of opposite sides. The preimages of the Weierstrass points are in this case the origin, the vertices (labeled $q_{i}$ on the right of Figure 1 and that are identified in the surface), and the hyperbolic midpoints of the sides (see right of Figure 1 where the midpoints are the $p_{i}$ ).
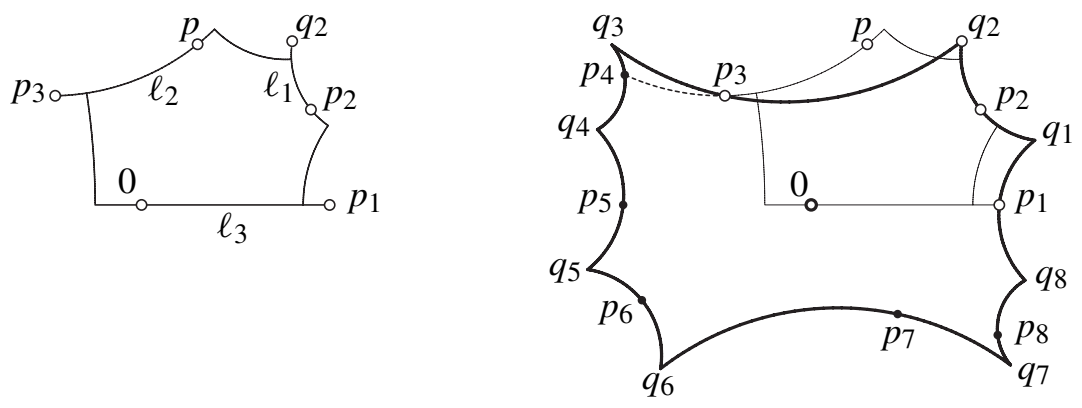

Figure 1

We want to associate such an octagon to a given set of Fenchel-Nielsen coordinates and vice-versa. We briefly recall the method of [Bu-Si2] to do this. Let $\left(2 \ell_{1}, t w_{1}, 2 \ell_{2}, t w_{2}, 2 \ell_{3}, t w_{3}\right)$, where the $\ell_{i}$ are lengths and the $t w_{i}$ are twist parameters, be Fenchel-Nielsen coordinates. Let $\mathscr{P}$ be a pair of pants with boundary lengths $2 \ell_{1}, 2 \ell_{2}, 2 \ell_{3}$. Cutting $\mathscr{P}$ along the geodesic arcs perpendicular to two boundary components we obtain two copies of a rectangular hexagon $\mathscr{H}$ with side lengths $\ell_{1}, \hat{\ell}_{3}$, $\ell_{2}, \hat{\ell}_{1}, \ell_{3}, \hat{\ell}_{2}$, where the $\hat{\ell}_{i}$ are the lengths of the perpendicular arcs.

Let $t_{i}=\ell_{i} \cdot t w_{i}$ and embed $\mathscr{H}$ isometrically in the unit disk with the edge of length $\ell_{3}$ on the real line, the first vertex at distance $t_{3}$ from the origin (to the left if $t_{3}$ is positive or to the right if it is negative) and let $p_{1}$ be on the real line at distance $\ell_{3}$ from the origin and at distance $t_{3}$ from the second vertex of the edge of length $\ell_{3}$. Shift the remaining vertices by $t_{1}$ and $t_{2}$ along the geodesics underlying the edges of lengths $\ell_{2}$ and $\ell_{3}$, using a similar sign convention, to obtain points $p_{2}, q_{2}, p$ and $p_{3}$ as illustrated on the left of Figure 1.

Let $h_{x}$ be the elliptic transformation of order 2 centered at point $x$. Then the remaining vertices of the octagon are constructed by means of the $h_{p_{i}}$ and $h_{0}$. Namely $q_{1}=h_{p_{2}}\left(q_{2}\right), q_{3}=h_{p_{3}}\left(q_{2}\right), q_{4}=h_{p_{4}}\left(q_{3}\right)$ and the remaining points are obtained by completing the construction of a symmetric octagon, i.e., taking images under $h_{0}$ (see right of Figure 1). 
The genus 2 surface $S$ is obtained from this octagon by identifying opposite sides, or in other words the Fuchsian group $G$ for this surface is generated by

$$
g_{1}=h_{p_{1}} \cdot h_{0}, \quad g_{2}=h_{p_{2}} \cdot h_{0}, \quad g_{3}=h_{p_{3}} \cdot h_{0} \quad \text { and } \quad g_{4}=h_{p_{4}} \cdot h_{0}
$$

The hyperbolic arcs,

$$
\left[q_{1}, q_{2}\right],\left[p_{3}, p_{4}\right] \cup\left[p_{8}, p_{7}\right] \text { and }\left[p_{5}, p_{1}\right] \text {, }
$$

define simple closed geodesics on the surface $S$ and together they define the pants decomposition we started with. Hence for this pants decomposition the surface has, by construction, Fenchel-Nielsen coordinates $\left(2 \ell_{1}, t w_{1}, 2 \ell_{2}, t w_{2}, 2 \ell_{3}, t w_{3}\right)$ where $t w_{i}=t_{i} / \ell_{i}$ (see [Bu-Si2] for more details).

The following important facts should be noted (see also [Ai et al.])

(i) The construction we have given will work for any set $\ell_{1}, \ell_{2}$ and $\ell_{3}$ in $\mathbb{R}^{+}$and any set $t w_{1}, t w_{2}$ and $t w_{3}$ in $\mathbb{R}$.

(ii) The octagon constructed is uniquely defined by the Fenchel-Nielsen coordinates.

(iii) Conversely starting from an octagon as above, one can compute the corresponding Fenchel-Nielsen coordinates by means of formulae given in [Bu], p. 454 and p. 38-39 (see [Bu-Si2]). In particular such an octagon defines a unique set of Fenchel-Nielsen coordinates for the pants decomposition defined by (1.2).

Another way of formulating this is that the construction yields a parameterization of the Teichmüller space of genus 2 surfaces by means of hyperbolic octagons.

We are now ready to introduce the elementary moves that we will elaborate upon in different contexts. These moves are half-twists, and their importance has been pointed out by Aline Aigon in [Ai]. We will do this in terms of octagons and in terms of the Fuchsian group, associated as above to the octagon.

Let $S$ be the surface $\mathbb{D} / G, G$ generated by the elements defined in (1.1), and let $\gamma$ be the closed geodesic image of the union of the geodesic arcs $\left[p_{3}, p_{4}\right]$ and $\left[p_{7}, p_{8}\right]$. Let $p_{3}^{\prime}$ be the hyperbolic midpoint of $\left[p_{3}, p_{4}\right]$ (see Figure 2) and $p_{4}^{\prime}=h_{p_{4}}\left(p_{3}^{\prime}\right)$. The surface $S^{\prime}$ obtained by performing a half twist along $\gamma$ has Fuchsian group generated by $g_{1}, g_{2}, g_{3}^{\prime}=h_{p_{3}^{\prime}} \cdot h_{0}$ and $g_{4}^{\prime}=h_{p_{4}^{\prime}} \cdot h_{0}$.

The fundamental octagon for $S^{\prime}$ is shown in Figure 2. It has vertices $q_{1}, q_{2}, q_{3}^{\prime}, q_{4}, q_{5}$, $q_{6}, q_{7}^{\prime}, q_{8}$, where $q_{3}^{\prime}=h_{p_{3}^{\prime}}\left(q_{2}\right)$, which is the same as $h_{p_{4}^{\prime}}\left(q_{4}\right)$, and $q_{7}^{\prime}=h_{p_{7}^{\prime}}\left(q_{6}\right)$. An informal formulation of this is that we have replaced the Weierstrass points defined by $p_{3}$ and $p_{4}$ by the midpoints of the arcs $\left[p_{3}, p_{4}\right]$ and $\left[p_{7}, p_{8}\right]$. 


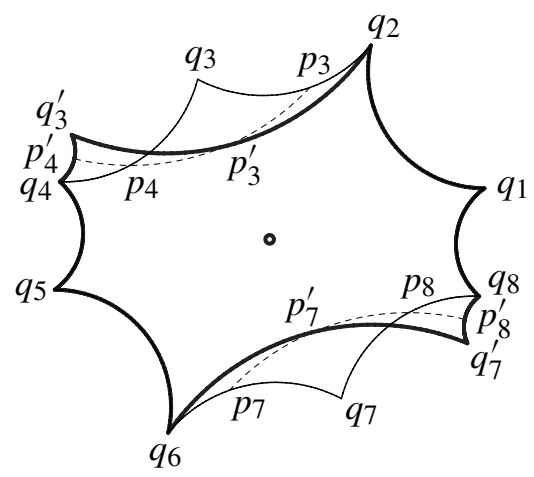

Figure 2

There are many other possibilities, but the description is basically the same (see [Ai] for a more complete treatment and [Si2] for other indications). This operation can also be formulated in terms of Fenchel-Nielsen coordinates. For the example we are considering it is,

$$
\left(\ell_{1}, t w_{1}, \ell_{2}, t w_{2}, \ell_{3}, t w_{3}\right) \mapsto\left(\ell_{1}, t w_{1}, \ell_{2}, t w_{2}+\frac{1}{2}, \ell_{3}, t w_{3}\right)
$$

In general there is no obvious algebraic relation between the equations for $S$ and $S^{\prime}$ but this will be the case if for example the point $p_{3}^{\prime}$ is a center of symmetry for the surface $S$. To make things more precise we need to introduce specific uniformizing functions.

For the first let $F$ be the $G$-equivariant meromorphic even function on the unit disk, two to one on the interior of the octagon $\backslash\{0\}$ and such that

$$
F(0)=0, \quad F\left(p_{1}\right)=1, \quad F\left(p_{3}\right)=\infty .
$$

Then an equation for $S$ is

$$
y^{2}=x(x-1)\left(x-F\left(q_{1}\right)\right)\left(x-F\left(p_{2}\right)\right)\left(x-F\left(p_{4}\right)\right)
$$

and $F$ is of course the uniformizing function yielding the $x$-coordinate (see [Bu-Si2]).

If $p_{3}^{\prime}$ is a center of symmetry for $S$ we will need a second form. Let $\varphi$ be the Möbius transformation sending 0 to $-1, F\left(q_{1}\right)$ to 1 and $F\left(p_{3}^{\prime}\right)$ to $\infty$. Write $F_{S}$ for the composed map $\varphi \circ F$. Then

$$
y^{2}=\left(x^{2}-1\right)\left(x^{2}-a\right)\left(x^{2}-b\right) \quad \text { where } a=F_{s}\left(p_{2}\right)^{2}, b=F_{s}\left(p_{4}\right)^{2}
$$

and again $F_{S}$ is a uniformizing function (see [Si2]). Note that in this context we have

$$
F_{S}(0)=-1, \quad F_{S}\left(q_{i}\right)=1, \quad F_{S}\left(p_{1}\right)=-F_{S}\left(p_{2}\right), \quad F_{S}\left(p_{3}\right)=-F_{S}\left(p_{4}\right)
$$

(see again $[\mathrm{Si} 2])$. 
In the case (1.6), that is when $p_{3}^{\prime}$ is a center of symmetry and more precisely $F_{s}\left(p_{3}^{\prime}\right)=\infty$, Aline Aigon [Ai] has shown that an equation for $S^{\prime}$ (obtained by a half-twist as in (1.2)) is

$$
y^{2}=\left(x^{2}-1\right)\left(x^{2}-a^{\prime}\right)\left(x^{2}-b^{\prime}\right) \quad \text { where } a^{\prime}=\frac{a(1-b)}{a-b}, b^{\prime}=1-b
$$

(see also for this form [Si2]).

\section{Genus 2 curves with an order 3 automorphism}

In this section we consider the one (complex) parameter family of genus 2 curves with an order 3 automorphism. The main result of this section is

2.1 Proposition. The transformations $\gamma_{1}:(\ell, t w, \ell, t w, \ell, t w) \mapsto\left(\ell, t w+\frac{1}{2}, \ell\right.$, $\left.t w+\frac{1}{2}, \ell, t w+\frac{1}{2}\right)$ and $\gamma_{2}$, induced by a rotation of a fundamental octagon (see Lemma 2.6 below), generate an action of $\mathrm{PSL}_{2}(\mathbb{Z})$ on the Teichmüller space of genus 2 surfaces with an order 3 automorphism. This action in turn induces an action of the symmetric group $\mathfrak{S}_{3}$ on a double cover of the moduli space of such surfaces.

There are various ways to describe the family of genus 2 curves with an order 3 automorphism, some probably simpler than others but for technical reasons we will use a less known way.

2.2 Lemma. Let $C$ be a genus 2 curve with an order 3 automorphism. Then an equation for $C$ can always be written in the form

$$
y^{2}=\left(x^{2}-t_{0}^{2}\right)\left(x^{2}-f_{3}\left(t_{0}\right)^{2}\right)\left(x^{2}-f_{3}\left(f_{3}\left(t_{0}\right)\right)^{2}\right)
$$

for some $t_{0} \neq 0, \pm 1, \pm 3, \pm i \sqrt{3}$ and where $f_{3}$ is

$$
f_{3}: t \mapsto \frac{3+t}{1-t} .
$$

Proof. If $C$ has an order 3 automorphism, then, by the classical classification of automorphism groups of genus 2 surfaces, its reduced group of automorphisms (the quotient of $\operatorname{Aut}(C)$ by the hyperelliptic involution) contains a dihedral group $D_{3}$. Let $f_{1}$, of order 2 , and $f_{2}$, of order 3 , generate such a dihedral group. Using a suitable conjugation we may assume that $f_{1}$ is $x \mapsto-x$. Since $f_{2}$ is of order 3 it is conjugate to $\left(\begin{array}{cc}j & 0 \\ 0 & j^{-1}\end{array}\right)$, with $j$ a third root of unity. But in this case the condition $-f_{2}^{2}(x)=f_{2}(-x)$ imposes that $f_{2}$ be of the form

$$
f_{2}: z \mapsto \frac{3 \alpha+z}{1-z / \alpha}, \quad \alpha \in \mathbb{C}, \alpha \neq 0 .
$$


Dividing the $x$-coordinate of the Weierstrass points by $\alpha$, we can choose $\alpha=1$ and hence $f_{2}=f_{3}$. To end the proof we note that the points $\pm t_{0}, \pm f_{3}\left(t_{0}\right)$ and $\pm f_{3}^{2}\left(t_{0}\right)$ are distinct unless $t_{0}=0, \pm 1, \pm 3, \pm i \sqrt{3}$.

An important fact motivating our choice is that

the orbit of 0 under $f_{3}$ is $0,3,-3$ and the orbit of infinity is $\infty,-1,1$.

Note that these points are fixed points of the involutions of the $D_{3}$ action.

In practice we will need a slightly different version of Lemma 2.2

2.4 Corollary. Let $C$ be a genus 2 curve with an order 3 automorphism. Then an equation for $C$ can always be written in the form

$$
y^{2}=x^{6}-(a+18) x^{4}+(2 a+81) x^{2}-a
$$

with $a \neq 0$ or -27 .

Moreover two curves $C$ and $C^{\prime}$ defined by a and $a^{\prime}$ are isomorphic if and only if $a^{\prime}=a$ or $a^{\prime}=729 / a$. More precisely the natural map from $\mathbb{C} \backslash\{0,-27\}$ to the coarse moduli space $\mathcal{M}_{2}$ of genus 2 curves is a double cover of its image ramified at $a=27$.

Proof. For the first part just take $a=\left(t_{0}+f_{3}\left(t_{0}\right)+f_{3}\left(f_{3}\left(t_{0}\right)\right)\right)^{2}$.

For the second assume first that the reduced automorphism group is $D_{3}$. In this case the only involutions of $C$ or $C^{\prime}$ are induced by transformations with two fixed points one in each of the orbits described in (2.3) and hence an isomorphism must be induced by a Möbius transformation preserving globally the points defined in (2.3). It is easily checked that this is only possible if the transformation is in the group generated by $x \mapsto-x, f_{3}$ and $x \mapsto 3 / x$. This yields $a^{\prime}=a$ or $a^{\prime}=729 / a$.

If the reduced automorphism group is larger than $D_{3}$ then it is of order 12, or 24 and there are only two such curves. The first, as is easily checked, corresponds to $a=27$. Hence the map $\varphi$ from $\mathbb{C} \backslash\{0,-27\}$ to the moduli space $\mathcal{M}_{2}$ of genus 2 curves is of degree 2 with ramification at the point for which $a=729 / a$ i.e., 27.

From the hyperbolic point of view the corresponding surfaces are also easy to describe. Start with a pair of pants with all three geodesic boundary components of equal hyperbolic lengths. Paste two copies of such a pair of pants in such a way that none of the geodesic boundaries are separating and with the same twist parameter for each. The order 3 symmetry of the pair of pants extends to a conformal order 3 automorphism of the resulting genus 2 surface. In terms of Fenchel-Nielsen coordinates these surfaces have coordinates of the form $(2 \ell, t w, 2 \ell, t w, 2 \ell, t w)$. Moreover since this order 3 automorphism is an homeomorphism, obviously non homotopic to the identity, it defines an element of the Teichmüller modular group, the fixed points of 
which are precisely the surfaces with the above Fenchel-Nielsen coordinates. Since in genus 2 there is only one topological type of order 3 automorphism the above defines a Teichmüller space for genus 2 surfaces with an order 3 automorphism.

In this context the hexagon used to build the octagon, as in Section 1, has side lengths $\ell, \hat{\ell}, \ell, \hat{\ell}, \ell, \hat{\ell}$ (notations similar to the ones used in Section 1). In particular it admits an order 3 symmetry.
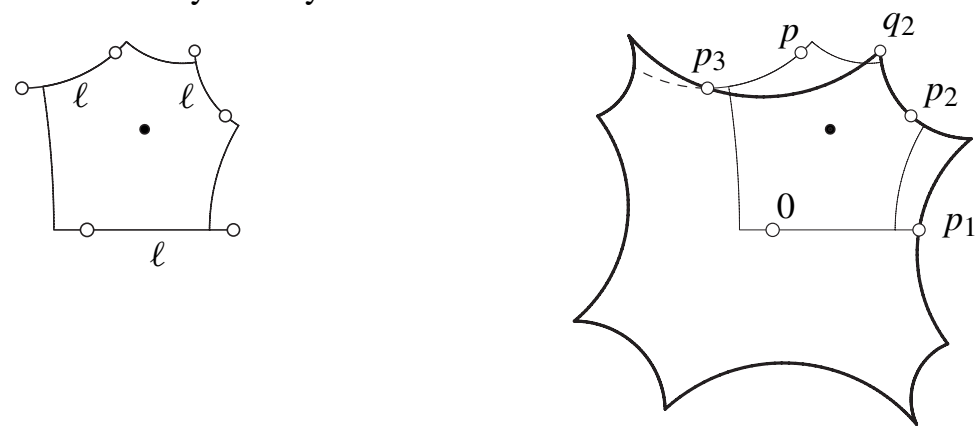

Figure 3

The order 3 automorphism being induced by the order 3 transformation on the hexagon one can easily deduce its action on the Weierstrass points (which are the images of 0 , the vertices and the midpoints of the sides). In particular if we denote by $\tilde{f}_{3}$ this transformation we have $\tilde{f}_{3}\left(p_{1}\right)=q_{2}, \tilde{f}_{3}\left(q_{2}\right)=p_{3}$ and $\tilde{f}_{3}\left(p_{3}\right)=p_{1}$.

2.5 Remark. By construction the Fenchel-Nielsen coordinates in $2 \ell$ and $t w$ correspond to the pants decomposition given in (1.2). On the other hand if we rotate the octagon in such a way that $p_{3}$ becomes a positive real we get new Fenchel-Nielsen coordinates. These correspond to the pants decomposition indicated in Figure 5. Noting this, it can easily be checked by following the action of $\tilde{f}_{3}$ (note that $p$, of Figure 3, is identified in the surface with $p_{8}$ and $p_{4}$ ) that the new Fenchel-Nielsen coordinates are again of the form $\left(2 \ell^{\prime}, t w^{\prime}, 2 \ell^{\prime}, t w^{\prime}, 2 \ell^{\prime}, t w^{\prime}\right)$.

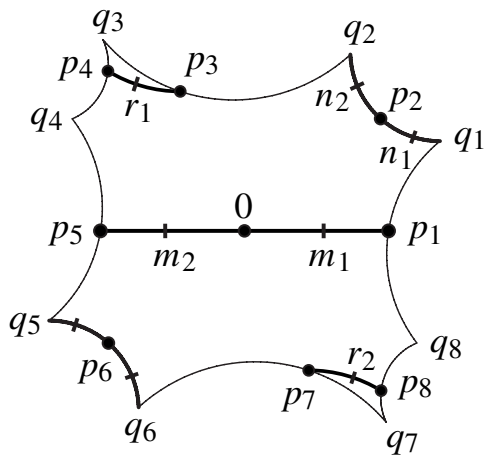

Figure 4

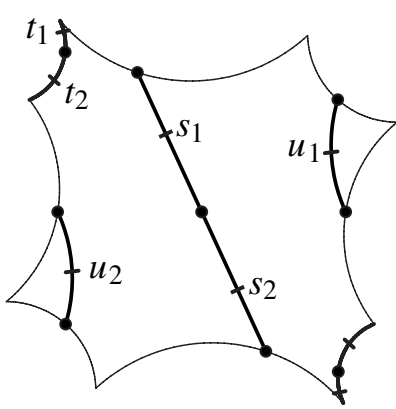

Figure 5 
2.6 Lemma. Let $(2 \ell, t w, 2 \ell, t w, 2 \ell, t w)$ correspond to the octagon of Figure 3 and let $\left(2 \ell^{\prime}, t w^{\prime}, 2 \ell^{\prime}, t w^{\prime}, 2 \ell^{\prime}, t w^{\prime}\right)$ correspond to the octagon obtained by the rotation indicated in Remark 2.5.

Let $L=\cosh (\ell), L^{\prime}=\cosh \left(\ell^{\prime}\right), T w=\cosh (t w \ell)$ and $T w^{\prime}=\cosh \left(t w^{\prime} \ell^{\prime}\right)$. We then have

$$
\begin{gathered}
L^{\prime}=T w^{2} \frac{2 L-1}{L-1}-1, \\
T w^{\prime}=\sqrt{\frac{T w^{2}(L+1)(2 L-1)-2\left(L^{2}-1\right)}{2 T w^{2}(2 L-1)-3(L-1)}}, \\
t w^{\prime}=-\operatorname{sign}(t w) \operatorname{arccosh}\left(T w^{\prime}\right) / \operatorname{arccosh}\left(L^{\prime}\right) .
\end{gathered}
$$

Proof. To find $\ell^{\prime}$ we only need, by Remark 2.5 , to compute the hyperbolic distance between 0 and $p_{3}$. For this note that in the construction we started with a rectangular hexagon with 3 sides of lengths $\ell$. The hyperbolic cosine of the remaining sides, i.e. $\cosh (\hat{\ell})$, is then $L /(L-1)$ (see $[\mathrm{Bu}]$ p. 454). We can now easily compute the length of $\left[0, p_{3}\right]$ using formula (2.3.2) in $[\mathrm{Bu}]$ p. 38. This yields $L^{\prime}$. The transformation being symmetric we can apply it twice and solve in $T w^{\prime}$ to recover $L$. Finally the reason for the sign change is the same as the one given in [OK].

Let, as in Section 1, the points $p_{i}$ be the midpoints of the sides of the octagon (see Figure 4). The fuchsian group $G$ is, as in (1.1), the group generated by $g_{i}=h_{p_{i}} h_{0}$, $1 \leqslant i \leqslant 4$, where as before $h_{x}$ is the order 2 elliptic transformation centered at the point $x$.

Let $u_{1}$ be the midpoint of [ $p_{1}, p_{2}$ ] (see Figures 4 and 5) and $r_{1}$ be the midpoint of $\left[p_{3}, p_{4}\right]$ (see Figure 4). Obviously we are in the situation described in (1.6) and we can use the uniformizing function $F_{s}$ for which we have in addition to the relations (1.7) the relations

$$
F_{S}\left(u_{1}\right)=0 \quad \text { and } \quad F_{S}\left(r_{1}\right)=\infty
$$

By Lemma 2.2, and its proof, we can easily find $t_{0}$ such that $t_{0} F_{s}\left(p_{3}\right)=f_{3}\left(t_{0}\right)$ and $t_{0} F_{s}\left(p_{1}\right)=f_{3}^{2}\left(t_{0}\right)$. Hence if we replace $F_{s}$ by $F_{1}=t_{0} F_{s}$ we have

$$
\begin{aligned}
& F_{1}\left(q_{i}\right)=-F_{1}(0)=t_{0} \\
& F_{1}\left(p_{3}\right)=-F_{1}\left(p_{4}\right)=f_{3}\left(t_{0}\right), \\
& F_{1}\left(p_{1}\right)=-F_{1}\left(p_{2}\right)=f_{3}\left(f_{3}\left(t_{0}\right)\right)
\end{aligned}
$$

(note that $p_{4}=-p_{8}$ and that $p=g_{3}\left(p_{8}\right)$ with $g_{3}$ as in (1.1)). In particular the algebraic curve has an equation of the form given in Lemma 2.2.

Let $m_{1}$ be the midpoint of [0, $\left.p_{1}\right], n_{1}$ the midpoint of $\left[q_{1}, p_{2}\right], r_{1}$ the midpoint of $\left[p_{3}, p_{4}\right]$ and so on as indicated in Figures 5 and 6. 
We still have $F_{1}\left(u_{1}\right)=0$, but $\tilde{f}_{3}\left(u_{1}\right)=g_{3}\left(-t_{2}\right)\left(g_{3}\right.$ as in (1.1) and $\tilde{f}_{3}$ as above) and $\tilde{f}_{3}\left(g_{3}\left(-t_{2}\right)\right)=m_{1}$, hence by $(2.3), F_{1}\left(t_{1}\right)=F_{1}\left(t_{2}\right)=3$ and $F_{1}\left(s_{1}\right)=F_{1}\left(s_{2}\right)=$ -3 . For the same reasons $F_{1}\left(m_{1}\right)=-1$ and $F_{1}\left(n_{1}\right)=1$.

2.7 Lemma. Let $G$ be, as above, the fuchsian group generated by $g_{1}, \ldots, g_{4}$. Let $\widetilde{G}$ be the extended group generated by $G, \tilde{f}_{3}, h_{0}$ and $h_{m_{1}}$. Then the quotient $\mathbb{D} / \widetilde{G}$ of the unit disk by this extended group is the Riemann sphere with four elliptic points, one of order 3 and three of order 2.

Proof. By construction the quotient of $\mathbb{D}$ by $G$ is a genus 2 surface $S$ with an automorphism group containing the dihedral group $D_{3}$. The quotient of $S$ by the hyperelliptic involution, which is induced by $h_{0}$, is the sphere with 6 marked points of order 2 . By the above we may assume that these points are $\pm t_{0}, \pm f_{3}\left(t_{0}\right)$ and $\pm f_{3}\left(f_{3}\left(t_{0}\right)\right)$. On this sphere $\tilde{f}_{3}$ induces $f_{3}$. Taking the quotient yields the sphere with 4 marked points, $b=\left(t_{0}+f_{3}\left(t_{0}\right)+f_{3}\left(f_{3}\left(t_{0}\right)\right)\right),\left(-t_{0}+f_{3}\left(-t_{0}\right)+f_{3}\left(f_{3}\left(-t_{0}\right)\right)\right)=-b$ and the images of the fixed points of $f_{3}, \pm 3 i \sqrt{3}$. On this quotient $h_{m_{1}}$ induces the same action as $h_{n_{1}}, h_{r_{1}}$ and so forth, in other words $h_{m_{1}}$ induces the transformation $x \mapsto-x$. Hence the final quotient is the sphere with the marked points $a=b^{2},-27$ of order 3 , and the images of the fixed points 0 and $\infty$. Note that the $a$ mentioned here is the same as the $a$ in Corollary 2.4. Note also that 0 is also the image in the last quotient of $s_{i}$, $u_{i}$ and $t_{i}$ while $\infty$ is also the image of $m_{i}, n_{i}$ and $r_{i}$.

Summarizing we have,

2.8 Corollary. Let $G$ and $\widetilde{G}$ be as in Lemma 2.7. Let

$$
y^{2}=x^{6}-(a+18) x^{4}+(2 a+81) x^{2}-a
$$

be an equation for the algebraic curve defined by $\mathbb{D} / G$. Then the quotient $\mathbb{D} / \widetilde{G}$ is the Riemann sphere with the four marked points

$$
-27 \text { of order } 3 \text { and } 0, \text { a and } \infty \text { of order } 2 .
$$

2.9 Proposition. Let $S$ be genus 2 surface with an order 3 automorphism. Let $(2 \ell, t w, 2 \ell, t w, 2 \ell, t w)$ be Fenchel-Nielsen coordinates for $S$ and let

$$
y^{2}=x^{6}-(a+18) x^{4}+(2 a+81) x^{2}-a
$$

be an equation for the corresponding algebraic curve.

Then the surface $S^{\prime}$ obtained by replacing $(\ell, t w)$ by $\left(\ell, t w+\frac{1}{2}\right)$ has equation

$$
y^{2}=x^{6}-\left(a^{\prime}+18\right) x^{4}+\left(2 a^{\prime}+81\right) x^{2}-a^{\prime} \quad \text { with } a^{\prime}=-(27+a)
$$

and the surface $S^{\prime \prime}$ obtained by replacing $(\ell, t w)$ by $\left(\ell^{\prime}, t w^{\prime}\right)$, with $\ell^{\prime}$ and $t w^{\prime}$ as defined in Lemma 2.6, has equation

$$
y^{2}=x^{6}-\left(a^{\prime \prime}+18\right) x^{4}+\left(2 a^{\prime \prime}+81\right) x^{2}-a^{\prime \prime} \quad \text { with } a^{\prime \prime}=729 / a .
$$


Proof. For notational reasons we prove the first assertion for $t w-\frac{1}{2}$ instead of $t w+\frac{1}{2}$. Since the transformation $a \mapsto a^{\prime}$ is an involution this is innocuous.

We are applying here simultaneously three half-twist along the non intersecting closed geodesics yielding the pants decomposition. The obvious generalization of the construction made in Section 1 shows that the group $G^{\prime}$ generated by

$$
g_{1}^{\prime}=h_{m_{1}} \cdot h_{m_{2}}, g_{2}^{\prime}=h_{n_{1}} \cdot h_{m_{2}}, g_{3}^{\prime}=h_{r_{2}^{\prime}} \cdot h_{m_{2}}, g_{4}^{\prime}=h_{r_{1}} \cdot h_{m_{2}}
$$

(where $r_{2}^{\prime}=g_{3}\left(r_{2}\right)$ ) is a fuchsian group for $S^{\prime}$ (see Figure 4 and Figure 6).

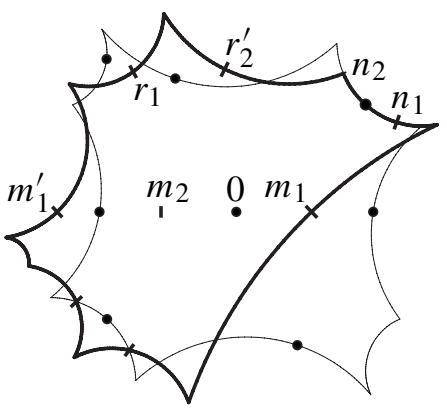

Figure 6

Writing $S^{\prime}=\mathbb{D} / G^{\prime}$ we note that, since $\tilde{f}_{3}\left(n_{2}\right)=m_{1}, \tilde{f}_{3}\left(n_{1}\right)=m_{2}$ and so forth, $\tilde{f}_{3}$ also induces an order 3 automorphism on $\mathbb{D} / G^{\prime}=S^{\prime}$. Also $h_{0}$ induces an involution, distinct from the hyperelliptic involution which is induced by $h_{m_{2}}$. Hence if $\widetilde{G}^{\prime}$ is the group generated by $G^{\prime}, h_{m_{2}}, h_{0}$ and $\tilde{f}_{3}$, then, as in Lemma $2.7, \mathbb{D} / \widetilde{G}^{\prime}$ is the sphere with the four marked points -27 (of order 3 ), $0, a^{\prime}$ and $\infty$.

On the other hand we obviously have $\widetilde{G}=\widetilde{G}^{\prime}$. Hence the two quotients are isomorphic the difference being that now $m_{i}, n_{i}$ and $r_{i}$ are sent to $a^{\prime}$, in place of $\infty$ while $0, p_{i}$ and $q_{i}$ are sent to $\infty$ in place of $a$. In other words there is a Möbius transformation taking $-27,0, a, \infty$ to $-27,0, \infty, a^{\prime}$, keeping -27 and 0 fixed and sending $a$ to $\infty$. It is $z \mapsto-\frac{(27+a) z}{z-a}$. The point $a^{\prime}$ being the image of $\infty$ is then equal to $-(27+a)$.

For the last assertion we note that if we replace the original octagon by the rotated octagon introduced in Lemma 2.6 we are replacing $p_{1}$ by $p_{3}, p_{2}$ by $p_{4}$ and so on. The role played by $u_{1}$ is now played by $r_{1}$ and it also exchanges the orbits of these two points. By (2.3) and the remarks made on $F_{1}$ the corresponding uniformizing function $F_{1}^{\prime}$ will be $3 / F_{1}$. In particular $t_{0}$ will be replaced by $3 / t_{0}$ (note that $3 / f_{3}(t)=$ $\left.f_{3}\left(f_{3}(3 / t)\right)\right)$ and $a$ by $729 / a$ (cf. Corollary 2.4$)$.

2.10 Lemma. Let $\gamma_{1}:(\ell, t w) \mapsto\left(\ell, t w+\frac{1}{2}\right)$ and $\gamma_{2}:(\ell, t w) \mapsto\left(\ell^{\prime}, t w^{\prime}\right), \ell^{\prime}$ and $t w^{\prime}$ as in Lemma 2.6. Then $\gamma_{2}{ }^{2}=1$ and $\left(\gamma_{1} \gamma_{2}\right)^{3}=1$. In particular they induce 
an action of $\mathrm{PSL}_{2}(\mathbb{Z})$ on the Teichmüller space of genus 2 surfaces with an order 3 automorphism.

Proof. It would be nice to have a direct geometric proof but unfortunately we have not as yet found one. Hence we proceed with an ugly computational proof. For this we first note that $\gamma_{2}^{2}=1$ is obvious, and that $\left(\gamma_{1} \gamma_{2}\right)^{3}=1$ is equivalent to $\gamma_{1} \gamma_{2} \gamma_{1}=\gamma_{2} \gamma_{1}^{-1} \gamma_{2}$. To express $\gamma_{2}, \gamma_{1}$ and $\gamma_{1}^{-1}$ in terms of $L$ and $T w$ (notations as in 2.6) we consider

$$
\begin{aligned}
& T_{2}=(L, T w) \mapsto\left(T w^{2} \frac{2 L-1}{L-1}-1, \sqrt{\frac{T w^{2}(L+1)(2 L-1)-2\left(L^{2}-1\right)}{2 T w^{2}(2 L-1)-3(L-1)}}\right), \\
& T_{1}=(L, T w) \mapsto\left(L, T w \sqrt{\frac{L+1}{2}}+\sqrt{T w^{2}-1} \sqrt{\frac{L-1}{2}}\right), \\
& T_{1}^{-1}=(L, T w) \mapsto\left(L, T w \sqrt{\frac{L+1}{2}}-\sqrt{T w^{2}-1} \sqrt{\frac{L-1}{2}}\right) .
\end{aligned}
$$

Because of the sign change indicated in Lemma 2.6, $\gamma_{1} \gamma_{2} \gamma_{1}$ will be represented by $T_{1}^{-1} T_{2} T_{1}$ and $\gamma_{2} \gamma_{1}{ }^{-1} \gamma_{2}$ by $T_{2} T_{1} T_{2}$. It can be proved by brute force that these are indeed equal but the computations involved, although reasonable enough for the length, turn out to be extremely heavy for the twist. On the other hand the computations simplify considerably for surfaces with zero twist i.e., with $T w=1$. We find fairly directly that $T_{2} T_{1} T_{2}$ maps $(L, 1)$ to

$$
\left(\frac{\left(2 L^{2}-L+1\right)}{2(L-1)}, \frac{1}{2} \sqrt{\frac{\left(2 L^{2}-3 L+3\right)(2 L-1)}{\left(L^{2}-L+1\right)(L-1)}}\right) .
$$

On the other hand $T_{1}^{-1} T_{2} T_{1}$ maps $(L, 1)$ to

$$
\left(\frac{\left(2 L^{2}-L+1\right)}{2(L-1)}, \frac{\sqrt{3+2 L^{3}-L^{2}} \sqrt{2 L^{2}+L-1}-\sqrt{(2 L-1)(L-1)^{2}} \sqrt{2 L^{2}-3 L+3}}{4 \sqrt{\left(L^{2}-L+1\right)(L-1)}}\right) .
$$

Squaring the numerator of the second term one easily finds that both transformations coincide if $T w=1$.

Remains to show that the sign condition is satisfied. For this we note that $\gamma_{2}(\ell, 0)=\left(\ell^{\prime}, 0\right)$ and $\gamma_{1}^{-1}\left(\ell^{\prime}, 0\right)=\left(\ell^{\prime \prime},-\frac{1}{2}\right)$. Hence $\gamma_{2} \gamma_{1}^{-1} \gamma_{2}(\ell, 0)$ will be of the form $\left(\ell^{\prime \prime \prime}, t w\right)$ with $t w \geqslant 0$. A value of $t w=\frac{1}{2}$ corresponds to $T w=\sqrt{\frac{L+1}{2}}$. Applying $T_{2}$ to this we have

$$
T_{2}\left(L, \sqrt{\frac{L+1}{2}}\right)=\left(\frac{(L+1)(2 L-1)}{2(L-1)}, \sqrt{\frac{3+2 L^{3}-L^{2}}{4\left(L^{2}-L+1\right)}}\right)
$$


from which it is easy to conclude that for the twist parameter $t w$ of $\gamma_{2} \gamma_{1}(\ell, 0)$ we will have $t w \geqslant-\frac{1}{2}$ and hence the twist parameter of $\gamma_{1} \gamma_{2} \gamma_{1}(\ell, 0)$ will be positive.

We conclude that $\gamma_{1} \gamma_{2} \gamma_{1}$ and $\gamma_{2} \gamma_{1}{ }^{-1} \gamma_{2}$ coincide on the full set of surfaces with zero twist, which is a subspace of real codimension 1 . On the other hand, by 2.10 , these transformations induce holomorphic maps on the parameter space $\mathbb{C} \backslash\{0,-27\}$. Since $\gamma_{1} \gamma_{2} \gamma_{1}$ and $\gamma_{2} \gamma_{1}{ }^{-1} \gamma_{2}$ are continuous bijections, this implies that they are holomorphic and we conclude that they coincide everywhere.

Proof of Proposition 2.1. The proposition follows from Lemma 2.10, Proposition 2.9 and the fact that $\sigma_{1}: a \mapsto-(a+27)$ and $\sigma_{2}: a \mapsto 729 / a$ generate an action of the symmetric group $\mathfrak{S}_{3}$ on the parameter space $\widehat{\mathbb{C}} \backslash\{-27,0, \infty\}$ which, by Corollary 2.4 , is a double cover of the moduli space of genus 2 curves with an order 3 automorphism.

2.12 Remark. It should be noted that although $\sigma_{2}$ operates trivially on the moduli space the action of $\sigma_{1} \sigma_{2}$ differs from the action induced by $\sigma_{1}$. To see this just note that $\sigma_{1} \sigma_{2}(a)=-27(a+27) / a$ is in general different from $-(a+27)$ or $-729 /(a+27)$ and apply Corollary 2.4 .

2.13 Examples. We limit here to examples that can immediately be deduced from the results of 2.9 .

- The fixed point of $\sigma_{2}$ is $a=27$. As noted earlier this is the curve with an automorphism group of order 24. It is easily checked that it corresponds to $\ell=$ $\operatorname{arccosh}(2), t w=0$, which is the fixed point of $\gamma_{2}$.

- The fixed point of $\sigma_{1}$ is $a=-\frac{27}{2}$. But $-\frac{27}{2}=\sigma_{2}\left(\sigma_{1}(27)\right)$, hence we can recover the Fenchel-Nielsen coordinates by applying $\gamma_{2} \gamma_{1}$ to the preceding case. We find $\ell=\operatorname{arccosh}\left(\frac{7}{2}\right)$ and $t w=\frac{1}{4}$.

- $\sigma_{1} \sigma_{2}$ has two fixed points, $-27 \frac{1 \pm i \sqrt{3}}{2}$, and these are exchanged by $\sigma_{2}$. To find the Fenchel-Nielsen coordinates we look for fixed points of $\gamma_{1} \gamma_{2}$. We find that $\ell=\operatorname{arccosh}(L)$, where $L$ is the solution $2.2057 \ldots$ of equation $8 x^{3}-12 x^{2}-$ $12 x-1=0$ and $t w=\frac{1}{4}$. Other considerations show that this in fact corresponds to $a=-27 \frac{1-i \sqrt{3}}{2}$. The other value for $a$ corresponds to the same value of $\ell$ but $t w=-\frac{1}{4}$. This is a fixed point of $\gamma_{1}^{-1} \gamma_{2}$. Both curves, with $t w= \pm \frac{1}{4}$, are of course isomorphic.

2.14 Remark. In this section we have only considered the pants decompositions indicated in Figure 4 and 5 but it is sometimes useful to consider other rotations of these.

An example of this is the following. Consider the surface defined by the coordinates $\left(2 \ell, \frac{1}{2}, 2 \ell, \frac{1}{2}, 2 \ell, \frac{1}{2}\right)$ and assume that we are using the pants decomposition (1.2). We can also use the pants decomposition defined by the $\operatorname{arcs}\left[p_{6}, p_{2}\right]$, 
$\left[p_{3}, p_{4}\right] \cup\left[p_{8}, p_{7}\right]$ and $\left[q_{8}, q_{1}\right]$. Elementary computations of the same type as the ones used in the proof of Lemma 2.6 show that the Fenchel-Nielsen coordinates are now $\left(2 \ell^{\prime}, \frac{1}{2}, 2 \ell, 0,2 \ell^{\prime}, \frac{1}{2}\right)$ where

$$
L^{\prime}=\cosh \left(\ell^{\prime}\right)=\frac{3 L-1}{2(L-1)} \quad \text { if } L=\cosh (\ell) .
$$

In particular we have $\ell^{\prime}=\ell$ if $L=(5+\sqrt{17}) / 4$. But in this case the associated algebraic curve is isomorphic to one of its transforms under the transformations indicated in (1.7). Looking how this isomorphism acts on the Weierstrass points one can show that the curve corresponds to $t_{0}=i \sqrt{10-2 \sqrt{17}} / 2$ and hence has an equation of type 2.4 with $a=-23+\sqrt{17}$.

\section{Genus 2 curves with an order 4 automorphism and cousins}

In this section we study two families. The first is the family of genus 2 surfaces with an order 4 automorphism. To formulate our main result concerning these we recall that from the hyperbolic point of view such surfaces are precisely the ones admitting a pants decomposition, with non-separating geodesics, and Fenchel-Nielsen coordinates of the form

$$
\left(2 \ell_{1}, t w, 2 \ell_{1}, t w, 2 \ell_{3}, \frac{1}{2}\right), \quad \text { with } \cosh \left(\ell_{3}\right)=2 \cosh \left(\ell_{1}\right)+1
$$

(see [Si2] but we will briefly indicate the construction below). With this we have

\subsection{Proposition. The transformations}

$$
\gamma_{1}:\left(2 \ell_{1}, t w, 2 \ell_{1}, t w, 2 \ell_{3}, \frac{1}{2}\right) \mapsto\left(2 \ell_{1}, t w+\frac{1}{2}, 2 \ell_{1}, t w+\frac{1}{2}, 2 \ell_{3}, \frac{1}{2}\right)
$$

and

$$
\gamma_{2}:\left(2 \ell_{1}, t w, 2 \ell_{1}, t w, 2 \ell_{3}, \frac{1}{2}\right) \mapsto\left(2 \ell_{1}^{\prime}, t w^{\prime}, 2 \ell_{1}^{\prime}, t w^{\prime}, 2 \ell_{3}^{\prime}, \frac{1}{2}\right),
$$

induced by a rotation of a fundamental octagon (see Lemma 3.9 below), generate an action of $\mathrm{PSL}_{2}(\mathbb{Z})$ on the Teichmüller space of genus 2 surfaces with an order 4 automorphism. This action in turn induces an action of the symmetric group $\mathfrak{S}_{3}$ on a double cover of the moduli space of such surfaces.

The second family is obtained by applying a half twist to the geodesic of length $2 \ell_{3}$. Namely we consider the sub-space of Teichmüller space defined by surfaces with Fenchel-Nielsen coordinates of the form $\left(2 \ell_{1}, t w, 2 \ell_{1}, t w, 2 \ell_{3}, 0\right)$, with $\cosh \left(\ell_{3}\right)=$ $2 \cosh \left(\ell_{1}\right)+1$. Denote by $\varphi$ the natural map

$$
\psi:\left(2 \ell_{1}, t w, 2 \ell_{1}, t w, 2 \ell_{3}, \frac{1}{2}\right) \mapsto\left(2 \ell_{1}, t w, 2 \ell_{1}, t w, 2 \ell_{3}, 0\right)
$$

For these surfaces the main result of this section is the following. 
3.3 Proposition. Surfaces with Fenchel-Nielsen coordinates of the form

$$
\left(2 \ell_{1}, t w, 2 \ell_{1}, t w, 2 \ell_{3}, 0\right) \text { with } \cosh \left(\ell_{3}\right)=2 \cosh \left(\ell_{1}\right)+1
$$

have equations of the form

$$
y^{2}=\left(x^{2}-1\right)\left(x^{2}-\alpha\right)(x-\alpha-1) \quad \text { with } \alpha \neq 0, \pm 1 .
$$

Moreover $\gamma_{1}$ and $\gamma_{2}$ (of Proposition 3.2) induces, via $\psi$, an action of $\mathrm{PSL}_{2}(\mathbb{Z})$ on the subspace of the genus 2 Teichmüller space defined by the above conditions and in turn this action defines an action of the symmetric group $\mathfrak{S}_{3}$ on the corresponding moduli space.

To prove these propositions we start by reviewing and reformulating some of the results of [Si2].

To this end let $\mathscr{H}$ be a rectangular hyperbolic hexagon with side lengths $\left(\ell_{1}, \ell_{2}, \ell_{1}, \ell_{2} / 2, \ell_{3}, \ell_{2} / 2\right)$, in that order (see left of Figure 7). Such hexagons exist, but the values of the $\ell_{i}$ are far from independent. In fact writing $L_{i}=\cosh \left(\ell_{i}\right)$ we have the relations

$$
L_{2}=\frac{L_{1}+1}{L_{1}-1} \quad \text { and } \quad L_{3}=2 L_{1}+1
$$

(see [Bu], p. 454).
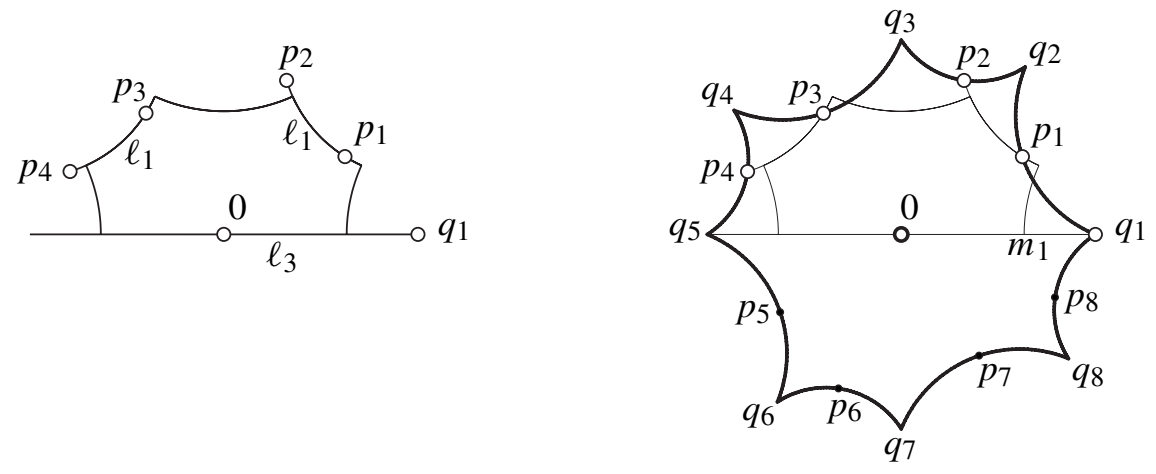

Figure 7

Embed isometrically this hexagon in the unit disk with the side of length $\ell_{3}$ on the real axis with midpoint at the origin. Let $q_{1}$ be positive real at hyperbolic distance $\ell_{3}$ from the origin. Then proceed as in Section 1 and shift the vertices of the edges of length $\ell_{1}$ by $t w \cdot \ell_{1}$ to obtain points $p_{1}$ to $p_{4}$ (see left of Figure 7).

To build the octagon from this data we let $p_{i+4}=-p_{i}, i=1, \ldots, 4$ and let $q_{i+1}=h_{p_{i}}\left(q_{i}\right), i=1, \ldots, 7$. 
Since the surface is obtained by identifying opposite sides of the octagon, the geodesic arcs

$$
\left[p_{1}, p_{2}\right] \cup\left[p_{6}, p_{5}\right], \quad\left[p_{3}, p_{4}\right] \cup\left[p_{8}, p_{7}\right] \quad \text { and } \quad\left[q_{5}, q_{1}\right]
$$

define simple closed geodesics. These in turn define a pants decomposition for which, by construction, the Fenchel-Nielsen coordinates are $\left(2 \ell_{1}, t w, 2 \ell_{1}, t w, 2 \ell_{3}, \frac{1}{2}\right)$.

Since the edge of the hexagon opposite to the one of length $\ell_{3}$ is twice the length of those opposite to those of length $\ell_{1}$ it is readily checked that the octagon we have constructed is stable under rotation by $\pi / 2$. In other words the surface has an order 4 automorphism. Moreover this automorphism being clearly non-homotopic to the identity it defines an element of the Teichmüller modular group, the fixed points of which are precisely the surfaces with Fenchel-Nielsen coordinates of form (3.1). Since, just as in the case of order 3 , there is only one topological type of automorphism of order 4, the Fenchel-Nielsen coordinates of (3.1) define a Teichmüller space for genus 2 surfaces with an order 4 automorphism.

By [Si2] (1.6) and (3.2) we have that the uniformizing map $F_{s}$ of (1.7) satisfies

$$
F_{S}\left(q_{1}\right)=-F_{S}(0)=1, \quad F_{S}\left(p_{2}\right)=-F_{S}\left(p_{1}\right)=a, \quad F_{s}\left(p_{4}\right)=-F_{S}\left(p_{3}\right)=1 / a .
$$

Hence an equation for the algebraic curve is,

$$
y^{2}=\left(x^{2}-a^{2}\right)\left(x^{2}-1\right)\left(x^{2}-1 / a^{2}\right) \quad \text { with } a \neq 0, \pm 1, \pm i .
$$

We also note that by [Si2] Section 3, since $m_{1}$ is the midpoint of 0 and $q_{1}$, we have $F_{s}\left(m_{1}\right)=-i$.

We now proceed to define the $\mathrm{PSL}_{2}(\mathbb{Z})$ action. For this let $\gamma_{1}$ be the transformation keeping the lengths fixed but replacing $t w$ by $t w+\frac{1}{2}$. The transformation induced on the equation is taken care of by

3.8 Proposition. Let $S$ be a genus 2 surface with an order 4 automorphism and Fenchel-Nielsen coordinates $\left(2 \ell_{1}, t w, 2 \ell_{1}, t w, 2 \ell_{3}, \frac{1}{2}\right)$ where $\cosh \left(\ell_{3}\right)=2 \cosh \left(\ell_{1}\right)+1$. Let

$$
y^{2}=\left(x^{2}-a^{2}\right)\left(x^{2}-1\right)\left(x^{2}-1 / a^{2}\right)
$$

be the associated equation (as in (3.7)).

Then the surface $S^{\prime}$ obtained by replacing $\left(\ell_{1}, t w\right)$ by $\left(\ell_{1}, t w+\frac{1}{2}\right)$ has equation

$$
y^{2}=\left(x^{2}+a^{2}\right)\left(x^{2}-1\right)\left(x^{2}+1 / a^{2}\right) .
$$

This is (3.2) of [Si2].

To define $\gamma_{2}$ we rotate the octagon so that $q_{2}$ becomes a positive real. This can equivalently be viewed as changing the pants decomposition. 
3.9 Lemma. Suppose that $S$ is a genus 2 surface with an order 4 automorphism and with Fenchel-Nielsen coordinates $\left(2 \ell_{1}, t w, 2 \ell_{1}, t w, 2 \ell_{3}, \frac{1}{2}\right)$ where $\cosh \left(\ell_{3}\right)=$ $2 \cosh \left(\ell_{1}\right)+1$. Suppose further that this set of coordinates corresponds to the octagon of Figure 7 and to the pants decomposition defined by $\left[p_{1}, p_{2}\right] \cup\left[p_{6}, p_{5}\right]$, $\left[p_{3}, p_{4}\right] \cup\left[p_{8}, p_{7}\right]$ and $\left[q_{5}, q_{1}\right]$. Then the Fenchel-Nielsen coordinates for the pants decomposition $\left[p_{2}, p_{3}\right] \cup\left[p_{7}, p_{6}\right],\left[p_{4}, p_{5}\right] \cup\left[p_{1}, p_{8}\right]$ and $\left[q_{6}, q_{2}\right]$ (see Figure 7 ) are of the form $\left(2 \ell_{1}^{\prime}, t w^{\prime}, 2 \ell_{1}^{\prime}, t w^{\prime}, 2 \ell_{3}^{\prime}, \frac{1}{2}\right)$ with again $\cosh \left(\ell_{3}^{\prime}\right)=2 \cosh \left(\ell_{1}^{\prime}\right)+1$. Moreover writing $L_{1}=\cosh \left(\ell_{1}\right), L_{1}^{\prime}=\cosh \left(\ell_{1}^{\prime}\right), T w=\cosh \left(t w \ell_{1}\right)$ and $T w^{\prime}=\cosh \left(t w^{\prime} \ell_{1}^{\prime}\right)$ we have

$$
\begin{aligned}
L_{1}^{\prime} & =T w^{2} \frac{2 L_{1}}{L_{1}-1}-1, \\
T w^{\prime} & =\sqrt{\frac{L_{1}^{2} T w^{2}+L_{1} T w^{2}-L_{1}^{2}+1}{2 L_{1} T w^{2}-L_{1}+1}}, \\
t w^{\prime} & =-\operatorname{sign}(t w) \operatorname{arccosh}\left(T w^{\prime}\right) / \operatorname{arccosh}\left(L_{1}^{\prime}\right) .
\end{aligned}
$$

Proof. We first note that the fact that the octagon is stable under rotation by $\pi / 2$ ensures that the new Fenchel-Nielsen coordinates are of the announced form. To compute these we note two facts. The first is that the midpoint of $\left[0, q_{2}\right]$ is also the midpoint of $\left[p_{1}, p_{2}\right]$. The second is that the hexagon we started with can be decomposed into four isometric copies of a trirectangular quadrangle and remaining angle $\pi / 4$ (at 0 ). With the formulae [Bu] p. 454 it is now easy to compute the length of the geodesic arc $\left[0, q_{2}\right]$. The hyperbolic cosine of this length is

$$
2 \frac{2 T w^{2} L_{1}}{L_{1}-1}-1
$$

But this is $2 L_{1}^{\prime}+1$ hence the value of $L_{1}^{\prime}$ found. Since the transformation is an involution we can solve it backwards to find $T w^{\prime}$. The sign condition on $t w^{\prime}$ is again the same as the one explained in $[\mathrm{Ok}]$.

We will denote by $\gamma_{2}$ the transformation that replaces $\left(\ell_{1}, t w\right)$ by $\left(\ell_{1}^{\prime}, t w^{\prime}\right)$ as defined in Lemma 3.9.

Starting with $\ell_{1}^{\prime}$ and $t w^{\prime}$ and associating an equation of the form (3.7), with the same method as before, will not lead to the same value for $a$. In fact as explained in [Si2] this yields the equation,

$$
y^{2}=\left(x^{2}-a^{\prime \prime 2}\right)\left(x^{2}-1\right)\left(x^{2}-1 / a^{\prime \prime 2}\right) \quad \text { with } a^{\prime \prime}=i \frac{i-a}{i+a} .
$$

Proof of Proposition 3.2. We first note that we again have $\gamma_{2}^{2}=1$ and $\left(\gamma_{1} \gamma_{2}\right)^{3}=1$. The proof is of the same nature as the proof of Lemma 2.10, and quite similar. 
Expressed in terms of $L$ and $T w$ both $\gamma_{1} \gamma_{2} \gamma_{1}$ and $\gamma_{2} \gamma_{1}{ }^{-1} \gamma_{2}$ transform $(L, 1)$ into

$$
\left(\frac{L^{2}+1}{L-1}, \sqrt{\frac{L\left(L^{2}-L+2\right)}{\left(L^{2}+1\right)(L-1)}}\right) .
$$

The rest of the argument is the same. Hence $\gamma_{1}$ and $\gamma_{2}$ induce an action of $\operatorname{PSL}_{2}(\mathbb{Z})$ on the Teichmüller space of genus 2 surfaces with an order 4 automorphism.

The way we have defined the transformations on the equations in Proposition 3.8 and in (3.10) is needed for the next part of this section. On the other hand the action of the permutation group $\mathfrak{S}_{3}$ is not clearly visible in this formulation. To recover this, simply write the equation in the form

$$
y^{2}=\left(x^{2}-1\right)\left(x^{4}+\mu x^{2}+1\right) \quad \text { with } \mu=-\left(a^{2}+1 / a^{2}\right) .
$$

Then the induced actions of $\gamma_{1}$ and $\gamma_{2}$ are given by

$$
\mu \mapsto-\mu \quad \text { and } \quad \mu \mapsto 2 \frac{\mu+6}{\mu-2}
$$

from which we recover the desired $\mathfrak{S}_{3}$ action.

Remains to show that the $\mu$-parameter space we have just introduced is a double cover of the moduli space of genus 2 curves with an order 4 automorphism. To do this recall that the automorphism group of a generic genus 2 curve with an order 4 automorphism is isomorphic to the dihedral group $D_{4}$ and the square of the order 4 automorphism is the hyperelliptic involution. If we write the equation of such a generic curve in the form (3.7), then the order 4 automorphisms are induced by $x \mapsto$ $1 / x$ and the non-hyperelliptic involutions are induced by $x \mapsto-x$ and $x \mapsto-1 / x$. From this we can conclude that, up to composition with automorphisms, the only Möbius transformations inducing isomorphisms are the identity and $x \mapsto \frac{i x+1}{x+i}$. The second possibility corresponds precisely to replacing $\mu$ by $\frac{2(\mu+6)}{\mu-2}$. Finally there are only two curves in the family which have an automorphisms group larger than $D_{4}$. The orders are 24, which corresponds to $\mu=1$ or $\mu=-14$ (see [Bu-Si1] (8.2)), and 48, which corresponds to $\mu=6$ (see [Bu-Si1] (8.13)). We conclude that our $\mu$-parameter space is a double cover of its image in moduli space ramified at $\mu=6$.

We now proceed to study the family introduced in Proposition 3.3. Since this family is obtained by applying a half twist to the first we are going to apply (1.8) to the present situation. For this we need to change octagons and consider the octagon associated by (1.2) to the coordinates $\left(2 \ell_{1}, t w, 2 \ell_{3}, \frac{1}{2}, 2 \ell_{1}, t w\right)$. In this case with the associated $F_{s}$ function we have equation

$$
y^{2}=\left(x^{2}-1\right)\left(x^{2}-a_{1}^{2}\right)\left(x^{2}-b_{1}^{2}\right) .
$$


To obtain $a_{1}$ and $b_{1}$ in terms of $a$ and $b$ we use the properties of $F_{s}$, indicated in Section 1, to compare where points are mapped in the curves defined by equations (3.7) and (3.11). Doing this we find that to pass from $(a, b)$ to $\left(a_{1}, b_{1}\right)$ we need a Möbius transformation that maps $-i$ to $\infty,-a$ to 1 and $1 / a$ to -1 . This is

$$
\varphi: z \mapsto \frac{a-i}{a+i} \cdot \frac{z-i}{z+i}
$$

In this context we have

$$
a_{1}=\varphi(a)=\frac{(i-a)^{2}}{(i+a)^{2}} \quad \text { and } \quad b_{1}=\varphi(1)=i \frac{i-a}{i+a} .
$$

Proof of Proposition 3.3. If we apply (1.8) to (3.12) we find that the algebraic curve associated to the surface with coordinates $\left(2 \ell_{1}, t w, 2 \ell_{3}, 0,2 \ell_{1}, t w\right)$ has for equation $y^{2}=\left(x^{2}-\alpha\right)\left(x^{2}-1\right)\left(x^{2}-\beta\right)$ with

$$
\alpha=\frac{a_{1}^{2}\left(1-b_{1}^{2}\right)}{a_{1}^{2}-b_{1}^{2}} \text { and } \beta=1-b_{1}^{2},
$$

but this just yields: $\alpha=a_{1}$ and $\beta=1+a_{1}$. This proves the first assertion of Proposition 3.3.

To end the proof of the proposition we will need the following

3.14 Lemma. Let $C$ and $C^{\prime}$ be the curves with respective equations $y^{2}=\left(x^{2}-\alpha\right)$. $\left(x^{2}-1\right)\left(x^{2}-\alpha-1\right)$ and $y^{2}=\left(x^{2}-\alpha^{\prime}\right)\left(x^{2}-1\right)\left(x^{2}-\alpha^{\prime}-1\right)$. Then $C$ is isomorphic to $C^{\prime}$ if and only if $\alpha^{\prime}=\alpha$ or $\alpha^{\prime}=1 / \alpha$.

Proof. We first note that if $\alpha^{\prime}=1 / \alpha$ then the two curves are clearly isomorphic. To see that the condition is also necessary consider two curves with equations $y^{2}=$ $\left(x^{2}-1\right)\left(x^{2}-\alpha\right)\left(x^{2}-\beta\right)$ and $y^{2}=\left(x^{2}-1\right)\left(x^{2}-\alpha^{\prime}\right)\left(x^{2}-\beta^{\prime}\right)$ and assume their automorphism groups do not contain elements of order 4 . Then the two curves are isomorphic if and only if $\left\{\alpha^{\prime}, \beta^{\prime}\right\}$ is, up to order, one of the six pairs

$$
\{\alpha, \beta\}, \quad\{1 / \alpha, 1 / \beta\}, \quad\{1 / \alpha, \beta / \alpha\}, \quad\{\alpha / \beta, 1 / \beta\}, \quad\{\alpha, \alpha / \beta\}, \quad\{\beta / \alpha, \beta\} .
$$

Replacing $\beta$ by $\alpha+1$ we obtain the result for curves without automorphisms of order 4.

If $C$ does have an automorphism of order 4 , replacing if necessary $\{\alpha, \beta\}$ by one of the pairs in (3.15), we may assume that $\beta=1 / \alpha$. In this case we must also consider the pairs obtained by applying (3.15) to

$$
\left\{-\left(\frac{\sqrt{\alpha}-i}{\sqrt{\alpha}+i}\right)^{2},-\left(\frac{\sqrt{\alpha}+i}{\sqrt{\alpha}-i}\right)^{2}\right\} .
$$


A case by case study shows that, up to isomorphism, the only pairs to consider are $\{\alpha, \alpha+1\}$ for $\alpha$ equal to $\frac{\sqrt{5}-1}{2},-\frac{\sqrt{5}+1}{2}$ or $\frac{i \sqrt{3}-1}{2}$ in which cases reexamining the lists one can directly show the result to be true.

End of Proof of Proposition 3.3. By Lemma 3.14 the map $\alpha \mapsto v=\alpha+1 / \alpha$ defines a map from $\mathbb{C} \backslash\{-1,0,1\}$ to moduli space. In terms of the parameter $a$ defining a curve with an order 4 automorphism in (3.7), we have by (3.12) and (3.13)

$$
v=\frac{2\left(\left(a^{2}-1\right)^{2}-4 a^{2}\right)}{\left(a^{2}+1\right)^{2}}
$$

Note that this expression for $v$ is invariant under $a \mapsto-a$ and $a \mapsto 1 / a$ and only depends on the equation (3.7).

We have seen that replacing $\left(\ell_{1}, t w\right)$ by $\left(\ell_{1}, t w+\frac{1}{2}\right)$ consists in replacing $a$ by $i a$ and replacing $\left(\ell_{1}, t w\right)$ by $\left(\ell_{1}^{\prime}, t w^{\prime}\right)$ consists in replacing $a$ by $\frac{i(i-a)}{i+a}$. Applying this to (3.16) we obtain the transformations

$$
\tau_{1}: v \mapsto 2 \frac{6-v}{2+v} \quad \text { and } \quad \tau_{2}: v \mapsto 2 \frac{v+6}{v-2} .
$$

Since $\tau_{1}$ and $\tau_{2}$ are of order 2 and $\tau_{1} \tau_{2}$ is of order 3 they generate a group isomorphic to $\mathfrak{S}_{3}$ and we are done.

3.18 Examples. We first look for fixed points for the transformations of (3.17).

- The fixed points of $\tau_{2}$ are -2 (which is excluded) and 6. This corresponds to $\alpha=3-2 \sqrt{2}$. The Fenchel-Nielsen coordinates for the corresponding surface where computed in [Bu-Si1], (8.3). We have $L_{1}=1+\sqrt{2}, t w=0$.

- The fixed points of $\tau_{1}$ are 2 (again excluded) and -6 . But this is the image under $\tau_{2} \tau_{1}$ of the preceding example. Hence to recover the Fenchel-Nielsen coordinates we only need to compute $\gamma_{2}\left(\gamma_{1}(1+\sqrt{2}, 0)\right)=(2+2 \sqrt{2},-1 / 4)$.

- Applying $\gamma_{1}$ to the first example we find the fixed point of $\tau_{2} \tau_{1} \tau_{2}$ which corresponds to $\alpha=i$ and of course to $L_{1}=1+\sqrt{2}, t w=\frac{1}{2}$.

- The fixed point of $\tau_{1} \tau_{2}$ corresponds, to $\alpha=i(\sqrt{3}-2)$. This is the curve associated to the last example of [Si2] hence $L_{1}=1+\sqrt{3}, t w=1 / 4$.

Also of interest are the curves in the family with larger automorphism groups. We have already encountered in the proof of Lemma 3.14 those with an order 4 automorphism.

- The case $\alpha=(\sqrt{5}-1) / 2$ was computed in [Bu-Si1] (8.1) it corresponds to $L_{1}=(1+\sqrt{5}) / 2, t w=0$.

- For $\alpha=-(1+\sqrt{5}) / 2$ we note that this can be obtained by applying $\tau_{2} \tau_{1} \tau_{2}$ to $\alpha^{\prime}+1 / \alpha^{\prime}$ with $\alpha^{\prime}=\sqrt{(\sqrt{5}-1) / 2}$. Hence to obtain the Fenchel-Nielsen coordinates 
we apply $\gamma_{2} \gamma_{1} \gamma_{2}$ to $((1+\sqrt{5}) / 2,0)$. This yields $L_{1}=(5+3 \sqrt{5}) / 2$ and $T w=$ $\sqrt{150+30 \sqrt{5}} / 10$. This curve was also considered in a different form in [Bu-Si1] (8.11).

- For $\alpha=(i \sqrt{3}-1) / 2$ we note that this corresponds to applying $\tau_{1}$ to $\alpha^{\prime}+1 / \alpha^{\prime}$ with $\alpha^{\prime}=(2-\sqrt{3})^{2}$. But $\alpha=7-4 \sqrt{3}$ is the second example of [Bu-Si1] (8.2), hence $L_{1}=3, t w=1 / 2$.

For those with an order 3 automorphism it is far more difficult to be systematic. We know nevertheless of two.

- The first corresponds to $L_{1}=4+\sqrt{17}, t w=\frac{1}{2}$. Assuming this corresponds to the pants decomposition (1.2), it has a second pants decomposition given by the $\operatorname{arcs}\left[q_{3}, q_{4}\right],\left[p_{6}, p_{2}\right]$ and $\left[p_{3}, p_{5}\right] \cup\left[p_{1}, p_{7}\right]$ all of length $2 \operatorname{arccosh}((5+\sqrt{17}) / 4)$. The twist parameters are $\frac{1}{2}, 0, \frac{1}{2}$. In other words this surface is isometric to the one considered in 2.14. As noted there it has an equation of the form given in Corollary 2.4 with $a=-23+\sqrt{17}$.

- The second corresponds to $L_{1}=(3+\sqrt{17}) / 4, t w=\frac{1}{2}$. But this is of the form (2.15) with $L=(5+\sqrt{17}) / 2$. Hence the order 3 automorphism by Remark 2.14 . Again an equation is easiest expressed in the form 2.4 with $a=\frac{-16767+729 \sqrt{17}}{512}$. Note that $\sigma_{2}$ of this value is $-23-\sqrt{17}$ (compare with the above).

\section{Quotients of genus 5 surfaces with an order 6 automorphism}

In this section we will prove parts of the statements of Theorem A and Theorem B. Namely the main result of this section is

4.1 Proposition. Let $S$ be a genus 2 surface with Fenchel-Nielsen coordinates $\left(2 \ell, t w, \ell, 2 t w, \ell^{\prime \prime}, 0\right)$, for a pants decomposition of type (1.2), and additional relation $\cosh \left(\ell^{\prime \prime} / 2\right)=2 \cosh (\ell / 2)$. Then the algebraic curve defined by $S$ has an equation of the form

$$
y^{2}=x(x-1)\left(x^{3}+a x^{2}-\frac{8}{3} a x+\frac{16}{9} a\right), \quad a \neq 0,-9 .
$$

Moreover the maps $\gamma_{1}:(\ell, t w) \mapsto\left(\ell, t w+\frac{1}{2}\right)$ and $\gamma_{2}:(\ell, t w) \mapsto\left(\ell_{2}, t w_{2}\right)$, with $\ell_{2}$ and $t w_{2}$ as in Theorem $B$, induce an action of $\mathrm{PSL}_{2}(\mathbb{Z})$ on the Teichmüller space of such surfaces. These transformations induce the maps $a \mapsto-9-a$ and $a \mapsto \frac{-9 a}{9+a}$ which define an action the symmetric group $\mathfrak{S}_{3}$ on a double cover of the moduli space of such surfaces. 
Before going into the proof of this proposition we will give a geometric description of the surfaces. We will consider dodecagons as fundamental domains in this section but revert to octagons in the next.

We start with a rectangular dodecagon $\mathscr{D}$ with edges alternatively of hyperbolic lengths $\ell$ and $\ell^{\prime}$ (see Figure 8). These lengths are of course related, in fact we have $\sinh (\ell / 2) \sinh \left(\ell^{\prime} / 2\right)=\sqrt{3} / 2$ (see [Bu], p. 454). Moreover if we call $\ell^{\prime \prime}\left(\right.$ resp. $\left.\ell^{\prime \prime \prime}\right)$ the length of the separating horizontal (resp. vertical) geodesic we have $\cosh \left(\ell^{\prime \prime} / 2\right)=$ $2 \cosh (\ell / 2)\left(\right.$ resp. $\left.\cosh \left(\ell^{\prime \prime \prime} / 2\right)=2 \cosh \left(\ell^{\prime} / 2\right)\right)$.

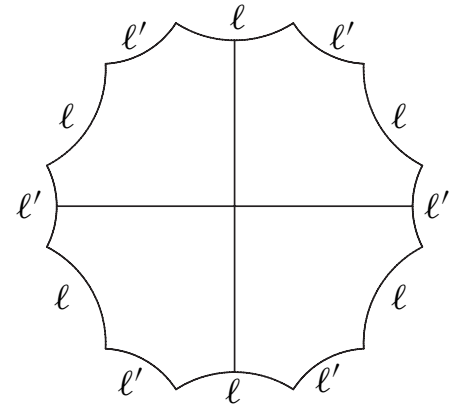

Figure 8

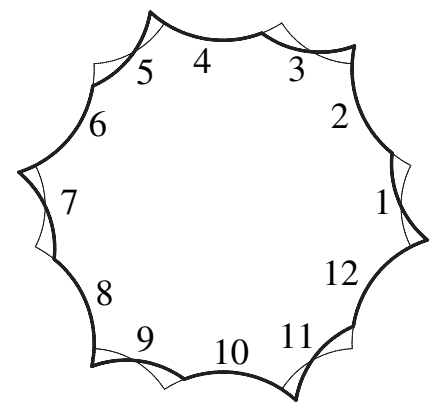

Figure 9

Paste two copies of the dodecagon $\mathscr{D}$ along the edges of length $\ell^{\prime}$. This yields a sphere with 6 disks removed and geodesic boundary components of length $2 \ell$. Now we can paste two copies of such a sphere to obtain a genus 5 surface $S_{1}$, which is hyperelliptic by its construction from two isometric spheres. We can do this last pasting with twist parameters, and if we do so with the same twist parameter $t w$ on the six boundary components we will then obtain a genus 5 surface with an order 6 automorphism (induced by rotation of angle $\pi / 3$ in the dodecagon). It also has involutions distinct from the hyperelliptic involution. In particular it is easy to construct a fixed point free involution $\varphi$ that exchanges the closed geodesics of length $2 \ell$, fixes globally two of length $2 \ell^{\prime}$ and exchanges two pairs of length $2 \ell^{\prime}$.

Since $\varphi$ is fixed point free the quotient $S_{2}=S_{1} / \varphi$ is of genus 3. Moreover from the construction of $\varphi$ we can give a geometric description of $S_{2}$ as follows. Consider a sphere with 4 disks removed and three geodesic boundaries of length $2 \ell$ and one of length $2 \ell^{\prime \prime}$. Then $S_{2}$ is obtained by pasting two copies of such a sphere using the twist parameter $t w$ (the same as above) on the geodesics of length $2 \ell$ and twist 0 on the one of length $2 \ell^{\prime \prime}$. From this it follows that $S_{2}$ is hyperelliptic. Moreover by construction it also admits non-hyperelliptic involutions.

One of these involutions is again fixed point free. The quotient of $S_{2}$ by this involution is of genus 2 with Fenchel-Nielsen coordinates $\left(2 \ell, t w, \ell, 2 t w, \ell^{\prime \prime}, 0\right)$. This defines the family of genus 2 surfaces we want to consider. 
There is a second description of the surfaces that will be useful. Start again with the dodecagon $\mathscr{D}$ but shift the end points of the edges of lengths $\ell$ by $t w \ell$ as shown in Figure 9. Then $S$ is obtain by the identifications

$$
1-7, \quad 2-12, \quad 3-5, \quad 4-10, \quad 6-8, \quad 9-11 .
$$

Note that this twisted dodecagon is stable under rotation by $\pi / 3$. The surface of genus 5 is obtained by pasting two copies of the twisted dodecagon to two copies of their mirror images. In this context the order 6 automorphism is induced by the $\pi / 3$ rotation.

Proof of Proposition 4.1. Since the genus 5 surface $S_{1}$ is hyperelliptic with an additional non-hyperelliptic involution $\varphi$ it has an equation of the form

$$
y^{2}=\prod_{i=1}^{6}\left(x^{2}-x_{i}^{2}\right),
$$

Moreover since it has an order 6 automorphism, we may assume by an argument similar to the one used in the proof of Lemma 2.2 that the $x_{i}$ are globally stable under the action of

$$
f: x \mapsto \frac{3 x-\sqrt{3}}{\sqrt{3} x+3}
$$

(note for further use that $f^{3}(t)=-1 / t$ and that $f(-t)=-f^{5}(t)$ ).

In this context the genus 3 quotient $S_{2}$ of $S_{1}$ by $\varphi$ has equation $y^{2}=x \prod_{i=1}^{6}\left(x-x_{i}^{2}\right)$. Let $y_{i}=\frac{x_{i}^{2}+1}{x_{i}^{2}-1}$. Relabeling the $y_{i}$ if necessary we obtain that $S_{2}$ has an equation of the form $y^{2}=\left(x^{2}-1\right) \prod_{i=1}^{3}\left(x^{2}-y_{i}^{2}\right)$. This change of coordinates for the Weierstrass points is exactly what is needed to recover $S$ simply or more precisely the genus 2 quotient $S$ of $S_{2}$ has for equation

$$
y^{2}=x(x-1) \prod_{i=1}^{3}\left(x-y_{i}^{2}\right) .
$$

Expanding (4.5) we obtain the equation (4.1.1) after a tedious but elementary computation.

The Fenchel-Nielsen coordinates in Proposition 4.1 correspond to the pants decomposition given by the arcs $(2-12) \cup(6-8),(4-10)$ and the horizontal axis in Figure 9.

We also have a second pants decomposition with the same properties, namely we can consider the pants decomposition defined by the arcs $(3-5) \cup(9-11),(1-7)$ and the line joining the midpoints of arc 4 to the midpoint of arc 10. Again this can 
be viewed as obtained by rotating the dodecagon. Applying the formulae in $[\mathrm{Bu}]$, p. 38-39 and 454 we can easily compute the Fenchel-Nielsen coordinates for this second decomposition. If we let $L=\cosh (\ell), T w=\cosh (t w \ell)$, then

$$
\begin{aligned}
L_{2} & =T w^{2} \frac{2 L+1}{L-1}-1, \\
T w_{2} & =\sqrt{\frac{2 T w^{2} L^{2}+3 T w^{2} L+T w^{2}-2 L^{2}+2}{4 T w^{2} L+2 T w^{2}-L+1}}, \\
t w_{2} & =-\operatorname{sign}(t w) \operatorname{arccosh}(T w) / \operatorname{arccosh}\left(L_{2}\right)
\end{aligned}
$$

and the Fenchel-Nielsen coordinates are $\left(2 \ell_{2}, t w_{2}, \ell_{2}, 2 t w_{2}, \ell_{2}^{\prime \prime}, 0\right)$, where $\cosh \left(\ell_{2}\right)=L_{2}$ and $\cosh \left(\ell_{2}^{\prime \prime} / 2\right)=2 \cosh \left(\ell_{2} / 2\right)$.

The quotient map from the genus 5 surface $S_{1}$ to the genus 2 surface $S$ is induced by the map

$$
\psi: x \mapsto \frac{\left(x^{2}+1\right)^{2}}{\left(x^{2}-1\right)^{2}} .
$$

Under this map the fixed points of $f$, which corresponds to $x= \pm i$ are mapped to the point with $x=0$. The fixed points of the non-hyperelliptic involutions of $S_{1}$ corresponds to the midpoints of the edges of the twisted Dodecagon of Figure 9. These correspond to $x$ either in the orbit of 0 under $f$ or in the orbit of 1. From our construction we may assume that $x=0$ corresponds to the point at the right end of the horizontal axis of Figure 9. Since by construction $f$ is induced by rotation of angle $\pi / 3$ in the dodecagon, this choice imposes that the midpoint of arc 4 corresponds to $x=-1$. Note for further use that $\psi(0)=1$ and $\psi(-1)=\infty$.

Let $G$ be the Fuchsian group generated by the identifications (4.2) and let $F_{D}$ be the even $G$-equivariant uniformizing function from the unit disk to the sphere such that $F_{D}$ sends 0 to 0 , sends the midpoint of the arc labeled 1 to 1 and the midpoint of the arc labeled 4 to infinity. By the above this means that this function $F_{D}$ is the uniformizing function giving the $x$-coordinate in the equation (4.1.1).

The function playing the role of the uniformizing function $F_{D}$ for the second pants decomposition (with coordinates defined by (4.6)) is clearly seen to be $z \mapsto$ $F_{D}(z) /\left(F_{D}(z)-1\right)$. Applying the map $x \mapsto x /(x-1)$ the equation (4.1.1) becomes

$$
y^{2}=x(x-1)\left(x^{3}+a_{2} x^{2}-\frac{8}{3} a_{2} x+\frac{16}{9} a_{2}\right) \quad \text { with } a_{2}=\frac{-9 a}{9+a}
$$

(cf. [Si1] (6.8)).

Now the only automorphism of a generic member of this family is the hyperelliptic involution. Hence the same argument as above shows that an isomorphism between two generic curves with equations of the form (4.1.1) must be induced by the identity or by a Möbius transformation leaving 0 fixed and exchanging 1 and $\infty$. But this is 
precisely how we have obtained $a_{2}$ in (4.8). This proves that the $a$-parameter space of (4.1.1) is a double cover of its image in moduli space, ramified at the point $a=-18$.

We again have $\gamma_{2}^{2}=1$ and $\left(\gamma_{1} \gamma_{2}\right)^{3}=1$. Unfortunately we do not have in this case either, a direct geometric proof. The only proof we know of, follows the same lines as the proof of Lemma 2.10 and is just as ugly. In terms of the $L$ and $T w$ introduced in (4.6) $\gamma_{1} \gamma_{2} \gamma_{1}$ and $\gamma_{2} \gamma_{1}{ }^{-1} \gamma_{1}$ transform $(L, 1)$ into

$$
\left(\frac{2 L^{2}+L+3}{2(L-1)}, \sqrt{\frac{4 L^{3}+9 L+5}{\left(L^{2}+L+1\right)(L-1)}}\right) .
$$

To end the proof of Proposition 4.1 we only need now

4.9 Lemma. Let $S$ be a surface with coordinates $\left(2 \ell, t w, \ell, 2 t w, \ell^{\prime \prime}, 0\right)$ and with $\cosh \left(\ell^{\prime \prime} / 2\right)=2 \cosh (\ell / 2)$. Let (4.1.1) be the associated equation for the surface.

Then the surface with Fenchel-Nielsen coordinates $\left(2 \ell, t w+\frac{1}{2}, \ell, 2 t w+1, \ell^{\prime \prime}, 0\right)$ has for equation

$$
y^{2}=x(x-1)\left(x^{3}+a_{1} x^{2}-\frac{8}{3} a_{1} x+\frac{16}{9} a_{1}\right) \quad \text { with } a_{1}=-9-a .
$$

Proof. Let as before $S_{1}$ be the genus 5 surface we have used in the construction of $S$. We also assume that $S_{1}$ has the equation considered in the proof of Proposition 4.1. Consider the group of automorphism of $S_{1}$ generated by the hyperelliptic involution, the automorphism induced by $f$ and the one induced by $x \mapsto-x$. The quotient of $S_{1}$ under this group is a sphere with 4 elliptic points one of order 6 , the image of the fixed points of $f$ and 3 of order 2 which are respectively the images of the Weierstrass points, the image of the points in the orbit under $f$ of the fixed points of $x \mapsto-x$ and finally the image of the points in the orbit under $f$ of the fixed points of $x \mapsto 1 / x$.

Now the map

$$
\varphi: x \mapsto-(\psi(x)+\psi(f(x))+\psi(f(f(x))))
$$

(with $\psi$ as in (4.7)) is a 12 to 1 map satisfying $\varphi(f(x))=\varphi(-x)=\varphi(x)$. In other words it induces the quotient map from $S_{1}$ to the sphere. The image of the Weierstrass points is of course $a$, the image of $i$ is 0 , while $\varphi(0)=-9$ and $\varphi(1)=\infty$.

Let $G$ be a Fuchsian group uniformizing $S_{1}$, and let $\widetilde{G}$ be the group generated by $G$ and elliptic transformations inducing $f$ and $x \mapsto-x$. The quotient of $\mathbb{D}$ by $\widetilde{G}$ is again the same sphere with the 4 marked points.

The same argument as the one used in the proof of Lemma 2.9 shows that replacing $t w$ by $t w+\frac{1}{2}$ in the construction of $S_{1}$ is equivalent to replacing $a$ by $\infty$ while keeping 0 and -9 fixed. This is achieved by $z \mapsto-(9+a) z /(z-a)$. Since $a_{1}$ is the image of $\infty$ under this map we obtain the result. Also since $a \mapsto a_{1}$ and $a \mapsto a_{2}$ clearly generate a group isomorphic to $\mathfrak{S}_{3}$, this also completes the proof of Proposition 4.1. 
4.11 Remarks. For use in the next section we will need three additional results on the surfaces studied in this section.

1) Consider again the genus 5 surface $S_{1}$ and its equation of the form (4.3). The midpoints of the arcs of length $\ell$ and $\ell^{\prime}$ have $x$ coordinate in the orbits of 1 and 0 under the function $f$ of (4.4). In particular in these orbits we have the points with $x$ coordinate $\sqrt{3}-2$ and $-1 / \sqrt{3}$. Applying the map $\psi$ of (4.7) to these points we obtain $4 / 3$ and 4. Let $m$ be the midpoint of the arc labeled 2 in Figure 9 and let $n$ be the midpoint of the arc labeled 3. From the geometric construction of the genus 2 quotient given at the beginning of this section we find that $F_{D}(m)=4 / 3$ and $F_{D}(n)=4$, where $F_{D}$ is the uniformizing function for the twisted dodecagon.

2) As noted earlier the twisted dodecagon of Figure 9 is globally invariant under rotation by $\pi / 3$. This rotation is of course incompatible with the identification of edges, of (4.2), used to define the genus 2 surface $S$. On the other hand we can construct a genus 3 double cover with an order 3 automorphism (this surface is distinct from the surface $S_{2}$ used in the construction of $S$ ). To do this consider the curve with equation

$$
y^{2}=P(x)=x(x+3)\left(x-t^{2}\right)\left(x-\left(f_{3}(t)\right)^{2}\right)\left(x-\left(f_{3}\left(f_{3}(t)\right)\right)^{2}\right)
$$

$f_{3}$ as in Lemma 2.2. If we apply the transformation $x \mapsto \frac{x}{3}+1$ to the roots of $P$ an elementary computation shows that the curves defined by (4.12) also have equations of the form given in (4.1.1). Hence (4.12) is just another description of the family.

But now the curve defined by

$$
y^{2}=\left(x^{2}+3\right)\left(x^{2}-t^{2}\right)\left(x^{2}-\left(f_{3}(t)\right)^{2}\right)\left(x^{2}-\left(f_{3}\left(f_{3}(t)\right)\right)^{2}\right)
$$

is a genus 3 double cover with an order 3 automorphism induced by $f_{3}$. We will use this in the next section.

3) We have shown that the image in moduli space of the family considered in this section is the quotient of $\widehat{\mathbb{C}} \backslash\{-9,0, \infty\}$ under $a \mapsto \frac{-9 a}{9+a}$. In particular it is isomorphic to the sphere minus two points and a cone point of order 2 (image of $a=-18$ ). Note also that the values $a=0,-9$ and $\infty$ correspond to singular curves and in particular the image is closed in moduli space.

4.14 Examples. As before we look for fixed points of the transformations. Up to isomorphy we have three fixed points. The first is given by $a=-18$ in equation (4.1.1) and was described in [Si1] 6.8. It corresponds to $\cosh (\ell)=1+\sqrt{3}, t w=0$. Note that in this case the curve has a larger automorphism group, in particular it has an automorphism of order 4, induced by $x \mapsto x /(x-1)$.

The second is defined by $a=-\frac{9}{2}$. This corresponds to $\gamma_{2} \gamma_{1}$ of the preceding. Hence to $\cosh (\ell)=1+\frac{\sqrt{3}}{2}$ and $t w=-\frac{1}{2}$. Note that this curve is isomorphic to the one defined by $a=9$, which corresponds to the transform by $\gamma_{1}$ of the first example. 
The third is given by $a=-\frac{9}{2}(1+i \sqrt{3})$, it corresponds to a fixed point of $\gamma_{1} \gamma_{2}$. This yields for $\cosh (\ell)$ the solution $3.1454 \ldots$ of equation $8 x^{3}-12 x^{2}-36 x-17$ and $t w=\frac{1}{4}$.

\section{Translation surfaces obtained from 3 squares}

In this section we complete the proof of Theorems A and B. This will be done by identifying the surfaces of Section 4 with surfaces in the $\mathrm{SL}_{2}(\mathbb{R})$ orbit of the translation surface tiled by 3 squares of the introduction.

But before that we need to recall a few facts. Let $C$ be a real genus 2 curve with three real components. Then it always admits an equation of the form,

$$
y^{2}=P(x)=x(x-1)\left(x-a_{1}\right)\left(x-a_{2}\right)\left(x-a_{3}\right) \quad \text { with } 1<a_{1}<a_{2}<a_{3} .
$$

Let $\gamma_{1}$ be the pull back in $C$ of $[0,1], \gamma_{2}$ the pull back of $\left[1, a_{1}\right]$ and so on up to $\gamma_{5}$ the pull back of $\left[a_{3}, \infty\right]$. Finally let $\gamma_{6}$ be the pull back of $[-\infty, 0]$. The $\gamma_{i}$ are simple closed curves in $C$ but to obtain cycles we need to orient them. For this we do the following.

Since $P$ is non zero in the upper half plane $\mathbb{H}$, and the latter is simply connected, we can choose on $\mathbb{H}$ a determination of the square root $\sqrt{P(x)}$. Obviously we can extend this determination to $\mathbb{R}$. We take the one which is positive on $[0,1]$. It will then be negative on $\left[a_{1}, a_{2}\right]$ and positive on $\left[a_{3}, \infty\right]$. It will also be pure imaginary with negative imaginary part on $\left[a_{1}, a_{2}\right]$, pure imaginary with positive imaginary part on $\left[a_{2}, a_{3}\right]$ and be pure imaginary with positive imaginary part on $[-\infty, 0]$.

With this determination of the square root, we can lift the natural orientation of $\mathbb{R}$ to a part of $\gamma_{i}$. We extend this orientation to $\gamma_{i}$. With this, the intersection numbers are now easy to compute they are $\gamma_{k} \cdot \gamma_{k+1}=1(k \bmod 6)$. As a consequence we have

$$
\gamma_{3}=-\gamma_{1}-\gamma_{5} \quad \text { and } \quad \gamma_{6}=-\gamma_{2}-\gamma_{4}
$$

We generalize this convention, let $a_{1}, a_{2}$ and $a_{3}$ be distinct complex numbers different from 0 and 1 . Let $\alpha_{1}$ be a simple arc in the complex plane joining 0 and 1 and not passing through any of the $a_{i}$. Let $\alpha_{2}$ join 1 and $a_{1}$ and not passing through the other $a_{i}$ and intersecting $\alpha_{1}$ only in 1 . Construct in the same way $\alpha_{3}$ from $a_{1}$ to $a_{2}$, $\alpha_{4}$ from $a_{2}$ to $a_{3}, \alpha_{5}$ from $a_{3}$ to $\infty$ and $\alpha_{6}$ from 0 to $\infty$ so the $\alpha_{i}$ only intersect in one point. Let $\gamma_{i}$ be the pull back in $C$ of $\alpha_{i}$. We can choose on each $\alpha_{i}$ a determination of the square root $\sqrt{P(x)}$ so that the induced orientation on the $\gamma_{i}$ is such that the intersection numbers are $\gamma_{k} \cdot \gamma_{k+1}=1(k \bmod 6)$. This is the convention we will use when dealing with both

$$
\int_{\gamma_{i}} \frac{a d x+b x d x}{y} \text { or } \int_{\alpha_{i}} \frac{a d x+b x d x}{\sqrt{P(x)}}
$$


Note that with this convention we again have (5.2).

5.4 Lemma. Let a be a real number, $a<-9$, and let $C$ be the curve defined by

$$
y^{2}=P_{a}(x)=x(x-1)\left(x^{3}+a x^{2}-\frac{8}{3} a x+\frac{16}{9} a\right) .
$$

Then for some real number $\lambda \neq 0,\left(C, \lambda \frac{x d x}{y}\right)$ is a translation surface in the $\mathrm{SL}_{2}(\mathbb{R})$ orbit of the translation surface tiled by 3 squares and holomorphic differential $d z$.

Proof. Let $C$ be the curve defined by $y^{2}=P_{a}(x)$. The conditions on $a$ are exactly the conditions for the roots of $P_{a}$ to be real and distinct. Let $\left(0,1, a_{1}, a_{2}, a_{3}\right)$ be the ordered set of roots of $P_{a}$. By the construction of the uniformizing map $F_{D}$ of Section $4, a_{1}$ will be the image under $F_{D}$ of the upper end point of arc 1 in Figure 9, $a_{2}$ the image of the upper endpoint of arc 2 and $a_{3}$ the upper end point of arc 3 .

By Remark 4.11,2) we can choose $t_{0}$ so that $a_{1}$ is mapped to $t_{0}^{2}$ by $x \mapsto 3(x-1)$, $a_{2}$ is mapped to $f_{3}\left(f_{3}\left(t_{0}\right)\right)^{2}$ and $a_{3}$ to $f_{3}\left(t_{0}\right)^{2}\left(f_{3}\right.$ as in Lemma 2.2).

On the curve $C_{1}$ defined by $w^{2}=z(z+3)\left(z-t_{0}^{2}\right)\left(z-f_{3}\left(t_{0}\right)^{2}\right)\left(z-f_{3}\left(f_{3}\left(t_{0}\right)\right)^{2}\right)$ the differential $\frac{x d x}{y}$ becomes, up to multiplication by $\sqrt{3}$,

$$
\omega_{1}=3 \frac{d z}{w}+\frac{z d z}{w}
$$

Let $C_{2}$ be the genus 3 double cover of $C_{1}$ defined by $w^{2}=\left(z^{2}+3\right)\left(z^{2}-\right.$ $\left.t_{0}^{2}\right)\left(z^{2}-f_{3}\left(t_{0}\right)^{2}\right)\left(z^{2}-f_{3}\left(f_{3}\left(t_{0}\right)\right)^{2}\right)$. The quotient map from $C_{2}$ to $C_{1}$ is defined by $(z, w) \mapsto\left(z^{2}, z w\right)$. From this it follows that the differential $\omega_{1}$ lifts to

$$
\omega_{2}=2\left(3 \frac{d z}{w}+\frac{z^{2} d z}{w}\right) .
$$

Now $C_{2}$ has the order 3 automorphism induced by $f_{3}$ and defined by

$$
\varphi_{3}:(z, w) \mapsto\left(\frac{3+z}{1-z}, \frac{16 w}{(1-z)^{4}}\right) .
$$

It is readily checked that $\omega_{2}$ is an invariant differential under this automorphism.

Let $\alpha$ be the simple closed geodesic in $C_{1}$ defined by arc 1 in Figure 9 . Since the midpoint of arc 1 is the point $(0,0)$ in $C_{1}, \alpha$ lifts to a simple closed geodesic $\alpha_{1}$ in $C_{2}$. Let $\alpha_{2}$ be the image of $\alpha_{1}$ under $f_{3}$. Similarly let $\beta$ be the simple closed geodesic in $C_{1}$ defined by arc 4 . Since the midpoint of arc 4 is the point at infinity of $C_{1}, \beta$ also lifts to a simple closed geodesic $\beta_{1}$ in $C_{2}$. Define $\beta_{2}$ in the same way as $\alpha_{2}$.

Since the differential $\omega_{2}$ is $\varphi_{3}$ invariant, we have

$$
\int_{\alpha_{1}} \omega_{2}=\int_{\alpha_{2}} \omega_{2} \text { and } \int_{\beta_{1}} \omega_{2}=\int_{\beta_{2}} \omega_{2} .
$$


The isomorphism between $C_{1}$ and $C$ is induced by $x \mapsto x / 3+1$, and by construction this map sends $t_{0}^{2}$ to $a_{1}, f_{3}\left(f_{3}\left(t_{0}\right)\right)^{2}$ to $a_{2}$ and $f_{3}\left(t_{0}\right)^{2}$ to $a_{3}$. Recalling the convention on the orientation of cycles and the choice of determination of $\sqrt{P_{a}(x)}$, we note that $x / \sqrt{P_{a}(x)}$ will be positive real on $[0,1]$, negative real on $\left[a_{1}, a_{2}\right]$, positive real on $\left[a_{3}, \infty\right]$, pure imaginary with negative imaginary part on $\left[1, a_{1}\right]$ and so forth.

Now the map from $C_{2}$ to $C_{1}$ is two to one on $\alpha_{1}$ and $\beta_{1}$. On the other hand $\alpha_{2}$ is mapped to the geodesic (6-8) $\cup(2-12)$ in $C_{1}$ and the covering map is one to one on $\alpha_{2}$. The same is true for $\beta_{2}$ which is mapped to (3-5) $\cup(9-11)$. Note that the Weierstrass points on the image of $\alpha_{2}$ are $\left(f_{3}\left(f_{3}\left(t_{0}\right)\right)^{2}, 0\right)$ and $\left(f_{3}\left(t_{0}\right)^{2}, 0\right)$ and those on the image of $\beta_{2}$ are $\left(t_{0}^{2}, 0\right)$ and $\left(f_{3}\left(f_{3}\left(t_{0}\right)\right)^{2}, 0\right)$.

Summarizing and using (5.2), we find that (5.7) translates into

$$
\begin{aligned}
& \int_{a_{1}}^{a_{2}} \frac{x d x}{\sqrt{P_{a}(x)}}=-2 \int_{0}^{1} \frac{x d x}{\sqrt{P_{a}(x)}}=-2 \int_{a_{3}}^{\infty} \frac{x d x}{\sqrt{P_{a}(x)}} \text { and } \\
& \int_{a_{2}}^{a_{3}} \frac{x d x}{\sqrt{P_{a}(x)}}=-2 \int_{1}^{a_{1}} \frac{x d x}{\sqrt{P_{a}(x)}}=-2 \int_{\infty}^{0} \frac{x d x}{\sqrt{P_{a}(x)}} .
\end{aligned}
$$

Letting $\omega=\frac{x d x}{y}$, the relations of (5.8) show that in terms of the locally flat metric defined by $\omega$, the points are the vertices of an L shaped polygon, as shown on the right of Figure 10, and that the surface $(S, \omega)$ is in the deformation space of the surface tiled by three squares and differential $d z$.
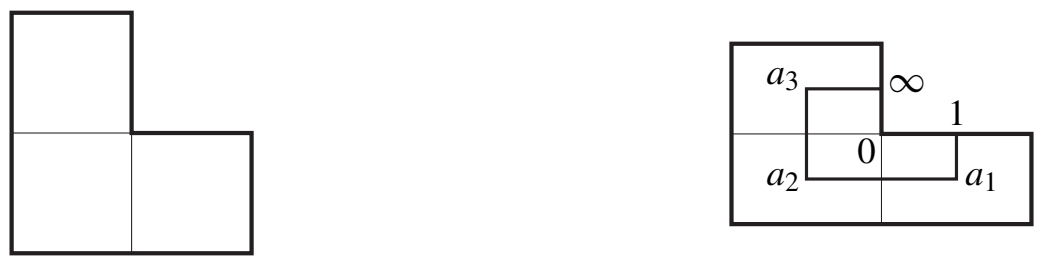

Figure 10

Proof of Theorem A. The image in moduli space of the $\mathrm{SL}_{2}(\mathbb{R})$-orbit of the surface tiled by three squares is the modular curve defined by the congruence sub-group generated by $\left(\begin{array}{ll}1 & 2 \\ 0 & 1\end{array}\right)$ and $\left(\begin{array}{cc}0 & -1 \\ 1 & 0\end{array}\right)$ (see [Hu-Le2] Remark 1.10). This is well known to be of genus 0 with two cusps and one elliptic point of order 2 . In particular as an algebraic curve it is smooth and irreducible.

On the other hand, by Remark 4.113) the moduli space of the surfaces considered in Section 4 is an algebraic curve, closed in the moduli space of genus 2 surfaces. By Proposition 4.1 and Lemma 5.4 the intersection of these two curves contains the isomorphy classes of the real curves with three real components in the family. Since this is of real codimension 1 the two curves in moduli coincide. 
To end the proof of Theorem A we only need to note that the differential $\omega$ has a double zero at the vertices of the $\mathrm{L}$ shaped polygon. In terms of equation (4.1.1) these vertices correspond to the point $(0,0)$. But this simply means that $\omega$ is a scalar multiple of $\frac{x d x}{y}$.

To prove Theorem B we are going to identify the transformations considered in Section 4 in the context of translation surfaces. For $\gamma_{2}$ of Proposition 4.1 this is relatively easy.

Consider the fundamental dodecagon for the surface. The circuit we have used to obtain the integrals of (5.8) is illustrated on the left of Figure 11.

If we let

$$
\alpha=\int_{0}^{1} \frac{x d x}{y} \text { and } \beta=\int_{1}^{a_{1}} \frac{x d x}{y}
$$

then by (5.8) the sequence of integrals along the arcs of the circuit will be $\alpha, \beta,-2 \alpha$, $-2 \beta, \alpha$ and $\beta$.

Now consider the circuit indicated on the right of Figure 11. Taking into account the identifications (4.2) we find that the values of the integrals along this circuit (starting again in 0 ) are

$$
-\beta, \quad \alpha, \quad 2 \beta, \quad-2 \alpha, \quad-\beta, \quad \alpha .
$$

But as explained in Section 4 the transformation $(\ell, t w) \mapsto\left(\ell_{2}, t w_{2}\right)$ of (4.10) corresponds precisely to the rotation bringing the point $p_{6}$ to the horizontal axis. Hence the transformation corresponds to $(\alpha, \beta) \mapsto(-\beta, \alpha)$. But the translation surface is built from three copies of the elementary parallelogram with vertices $0,2 \alpha, 2 \alpha+2 \beta, 2 \beta$ and the transformation is obtained by applying $\left(\begin{array}{cc}0 & -1 \\ 1 & 0\end{array}\right)$
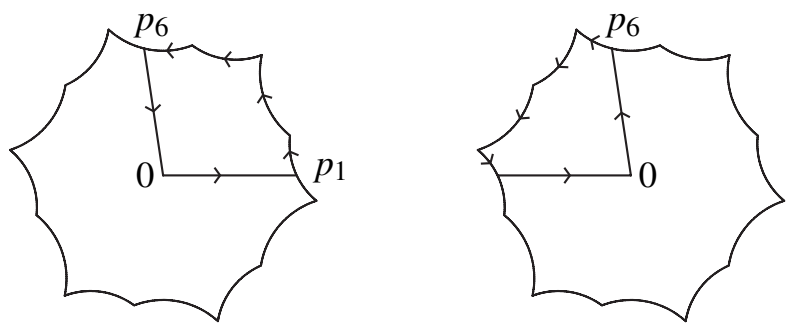

Figure 11

The description of the transformation $(\ell, t w) \mapsto\left(\ell, t w+\frac{1}{2}\right)$ is a little more involved. To make the arguments easier to follow we are going to change models on both sides. For the translation surface this is easy we cut two of the parallelograms forming the $\mathrm{L}$ shaped polygon and re-paste using the identifications. We obtain in this 
way a star shaped polygon as illustrated on the right of Figure 12. The identifications are now by opposite sides.

For the hyperbolic model, we use the octagon with Fenchel-Nielsen coordinates $\left(\ell^{\prime \prime}, 0, \ell, 2 t w, 2 \ell, t w\right)$ and pants decomposition (1.2).
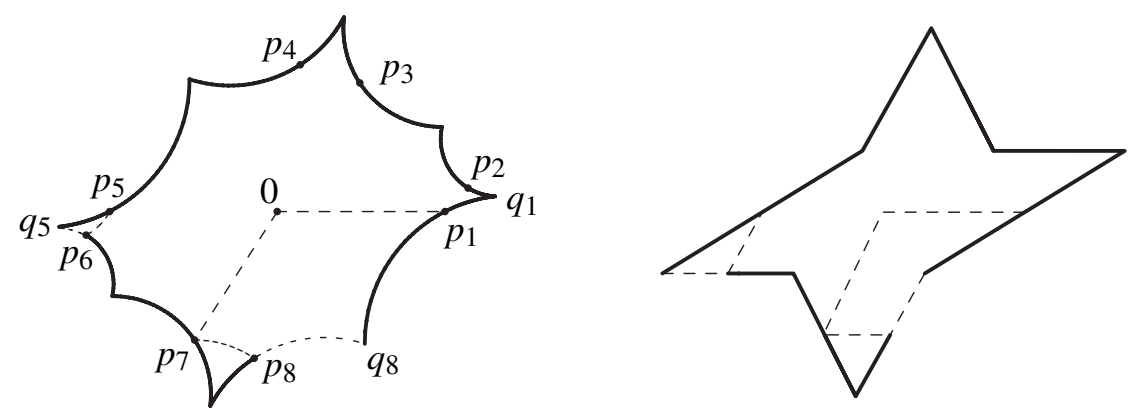

Figure 12

Now consider the uniformizing function $F$ of (1.4). Let $b_{1}=F\left(p_{2}\right), b_{2}=F\left(q_{1}\right)$ and $b_{3}=F\left(p_{4}\right)$ (see Figure 12). In general the $b_{i}$ are distinct from the roots $a_{i}$ of the polynomial of Theorem A. Let $F_{1}$ be $F$ composed with

$$
z \mapsto \frac{b_{1}-b_{3}}{b_{1}-b_{2}} \cdot \frac{z-b_{2}}{z-b_{3}} .
$$

Then

$$
F_{1}\left(q_{i}\right)=0, \quad F_{1}\left(p_{3}\right)=F_{1}\left(p_{6}\right)=1 \quad \text { and } \quad F_{1}\left(p_{4}\right)=F_{1}\left(p_{8}\right)=\infty .
$$

Moreover comparing lengths one can check that

$$
F_{1}(0)=a_{2}, \quad F_{1}\left(p_{1}\right)=F_{1}\left(p_{5}\right)=a_{1} \quad \text { and } \quad F_{1}\left(p_{3}\right)=F_{1}\left(p_{7}\right)=a_{3} .
$$

In particular the integration circuit we have been using is the one illustrated by doted arcs on the left of Figure 12 and starts at $q_{5}$.

We want now to describe the transformation

$$
\left(\ell^{\prime \prime}, 0, \ell, 2 t w, 2 \ell, t w\right) \mapsto\left(\ell^{\prime \prime}, 0, \ell, 2 t w+1,2 \ell, t w+\frac{1}{2}\right)
$$

in terms of octagons and Fuchsian groups.

We split the transformation (5.13) in two. In the first step we transform the initial octagon into the one for the coordinates $\left(\ell^{\prime \prime}, 0, \ell, 2 t w+1,2 \ell, t w\right)$. This is shown on the left of Figure 13. We complete by the action of a half twist along the horizontal geodesic $\left[p_{5}, p_{1}\right]$ to obtain an octagon representing $\left(\ell^{\prime \prime}, 0, \ell, 2 t w+1,2 \ell, t w+\frac{1}{2}\right)$ (see the right of Figure 13). 

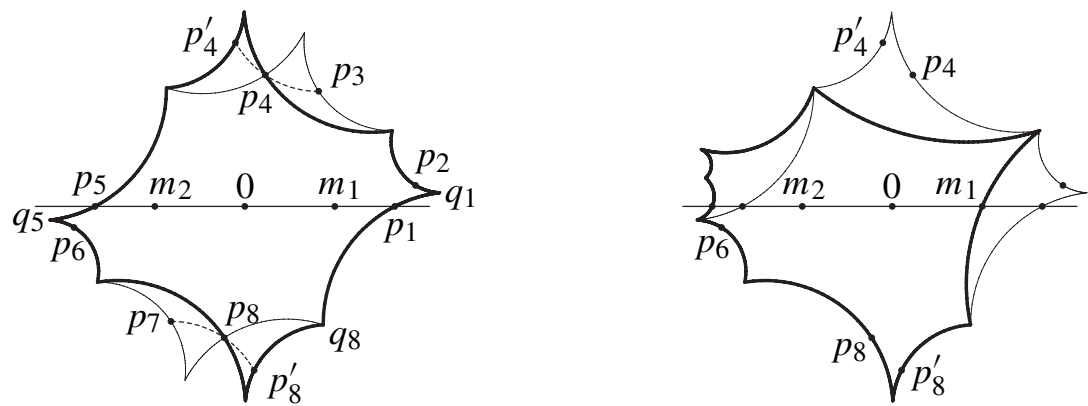

Figure 13

Call $G$ the group generated by,

$$
g_{1}=h_{0} \cdot h_{p_{5}}, \quad g_{2}=h_{0} \cdot h_{p_{6}}, \quad g_{3}=h_{0} \cdot h_{p_{7}}, \quad g_{4}=h_{0} \cdot h_{p 8}
$$

Where as before $h_{p}$ is the elliptic transformation of order 2 centered at $p$. Let $p_{8}^{\prime}=h_{p_{8}}\left(p_{7}\right)$ and let

$$
g_{4}^{\prime}=h_{0} \cdot h_{p_{8}^{\prime}}, \quad g_{2}^{\prime \prime}=h_{m_{2}} \cdot h_{p_{6}}, \quad g_{3}^{\prime \prime}=h_{m_{2}} \cdot h_{p_{8}}, \quad g_{4}^{\prime \prime}=h_{m_{2}} \cdot h_{p_{8}^{\prime}}
$$

Call $G^{\prime}$ the group generated by $g_{1}, g_{2}, g_{4}$ and $g_{4}^{\prime}$ and call $G^{\prime \prime}$ the group generated by $g_{1}, g_{2}^{\prime \prime}, g_{3}^{\prime \prime}$ and $g_{4}^{\prime \prime}$. The groups $G, G^{\prime}$ and $G^{\prime \prime}$ are the groups identifying the opposite edges of the three octagons of Figure 13.

Let $C$ be the algebraic curve with equation as in Theorem A and associated to the hyperbolic surface by Proposition 4.1. The function $F_{1}$ we have introduced in (5.11) and (5.12) is the uniformizing function for the group $G$, giving the $x$-coordinate. Let $\pi_{1}$ be the $G$-uniformizing map from the unit disk to $C$ such that the $x$-coordinate of $\pi_{1}(z)$ is $F_{1}(z)$ and such that the induced orientation on the arcs along which we integrate coincides with the one we have used above.

5.14 Lemma. Let

$$
\omega=\pi_{1}^{*}\left(\frac{x d x}{y}\right) .
$$

Then $\omega$ is an invariant differential for the three groups $G, G^{\prime}$ and $G^{\prime \prime}$.

Proof. For $G$ this is by definition. For $G^{\prime}$ all we need to note is that $p_{4}^{\prime}$ being in the orbit of $p_{8}$ under $G, h_{p_{8}^{\prime}}$ induces the hyperelliptic involution. Hence $h_{p_{8}^{\prime}}{ }^{*}(\omega)=-\omega$. Since $h_{0}$ acts in the same way we have the invariance under $g_{4}^{\prime}$.

We have $F_{1}(0)=a_{2}$ and $F_{1}\left(p_{1}\right)=F_{1}\left(p_{5}\right)=a_{1}$. On the other hand $m_{1}$ and $m_{2}$ are the midpoints of $\left[0, p_{1}\right]$ and $\left[p_{5}, 0\right]$. By Remark 4.111$)$ we conclude that $F_{1}\left(m_{1}\right)=F_{1}\left(m_{2}\right)=4 / 3$. 
Now consider the curve $C_{2}$ of genus 3 defined in Remark 4.112 ) and used in the proof of Lemma 5.4 and let $\varphi: C_{2} \rightarrow C$ be the map defined by

$$
\varphi:(x, y) \mapsto\left(\frac{x^{2}}{3}+1, \frac{x y}{9 \sqrt{3}}\right) .
$$

Note for further use that the image of the points with $x$-coordinate \pm 1 in $C_{2}$ are the points with $x$-coordinate $4 / 3$ in $C$.

Let $\pi_{2}$ be the map from the unit disk to $C_{2}$ such that $\varphi \cdot \pi_{2}=\pi_{1}$. From the above we obtain that $\pi_{2}\left(m_{2}\right)$ is a point with $x$-coordinate \pm 1 in $C_{2}$.

Let $f:(x, y) \mapsto(-x, y)$ in $C_{2}$ and let $\omega_{2}=\varphi^{*}\left(\frac{x d x}{y}\right)$. Up to multiplication by a scalar this is the same as the $\omega_{2}$ of (5.5) and from this we obviously have $f^{*}\left(\omega_{2}\right)=$ $-\omega_{2}$. Also since $\omega_{2}$ is $\varphi_{3}$ invariant $\left(\varphi_{3}\right.$ as in (5.6)) we have $\left(\varphi_{3}^{-1} f \varphi_{3}\right)^{*}\left(\omega_{2}\right)=-\omega_{2}$. But since $\pi_{2}\left(m_{2}\right)$ is a fixed point of $\varphi_{3}^{-1} f \varphi_{3}$, this involution is induced by $h_{m_{2}}$. Hence $h_{m_{2}}^{*}\left(\pi_{2}^{*}\left(\omega_{2}\right)=-\pi_{2}^{*}\left(\omega_{2}\right)\right.$ or since by definition of $\omega_{2}$ and $\pi_{2}, \pi_{2}^{*}\left(\omega_{2}\right)=\omega$, we have

$$
h_{m_{2}}^{*}(\omega)=-\omega .
$$

With the same argument as for $G^{\prime}$ we conclude that $\omega$ is $G^{\prime \prime}$ invariant.

If

$$
\alpha=\int_{q_{5}}^{p_{6}} \omega \quad \text { and } \quad \beta=\int_{p_{6}}^{p_{5}} \omega
$$

then by (5.8), (5.11) and (5.12) we also have

$$
\int_{p_{1}}^{0} \omega=-2 \alpha, \quad \int_{0}^{p_{7}} \omega=-2 \beta, \quad \int_{p_{7}}^{p_{8}}=\alpha, \quad \int_{p_{8}}^{q_{8}} \omega=\beta .
$$

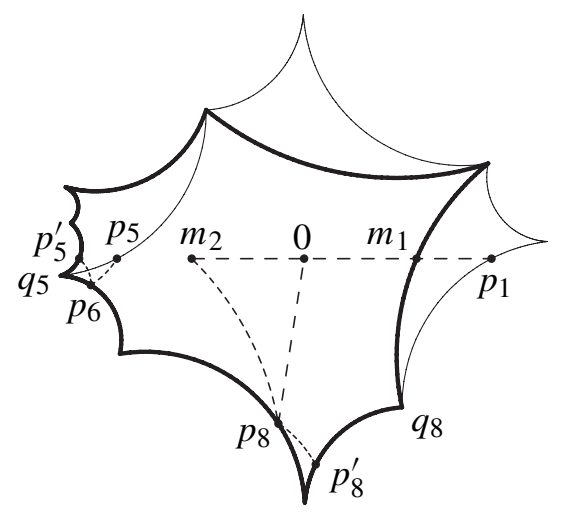

Figure 14 
5.15 Lemma. We have

$$
\int_{p_{6}}^{p_{5}^{\prime}} \omega=\beta-\alpha, \int_{m_{1}}^{m_{2}} \omega=-2 \alpha, \int_{m_{2}}^{p_{8}} \omega=2 \alpha-2 \beta, \int_{p_{8}}^{p_{8}^{\prime}} \omega=\alpha, \int_{p_{8}^{\prime}}^{q_{8}} \omega=\beta-\alpha
$$

(see Figure 14).

Proof. The arguments used in the proofs of Lemma 5.4 and Lemma 5.14 show that

$$
\int_{m_{1}}^{0} \omega=\int_{0}^{m_{2}} \omega=\int_{m_{2}}^{p_{5}} \omega=\int_{p_{5}}^{p_{5}^{\prime}} \omega=\frac{1}{2} \int_{p_{1}}^{0} \omega=-\alpha .
$$

This proves the assertion about the integral between $m_{1}$ and $m_{2}$. Moreover since we clearly have

$$
\int_{p_{6}}^{p_{5}^{\prime}} \omega=\int_{p_{6}}^{p_{5}} \omega+\int_{p_{5}}^{p_{5}^{\prime}} \omega=-\alpha+\beta
$$

the assertion for the integral between $p_{6}$ and $p_{5}^{\prime}$ is also proved.

For obvious reasons (see Figures 13 and 14) we have

$$
\int_{p_{8}}^{p_{8}^{\prime}} \omega=\int_{p_{7}}^{p_{8}} \omega, \quad \int_{m_{2}}^{p_{8}} \omega=\int_{0}^{p_{8}} \omega-\int_{0}^{m_{2}} \omega \quad \text { and } \quad \int_{p_{8}^{\prime}}^{q_{8}} \omega=\int_{p_{8}}^{q_{8}} \omega-\int_{p_{7}}^{p_{8}} \omega .
$$

This proves the remaining three assertions of the lemma.

Calling $S$ the surface $\mathbb{D} / G$ an $S^{\prime \prime}$ the surface $\mathbb{D} / G^{\prime \prime}$ we have shown that the values of the integrals of $\omega$ along the circuits are respectively

$$
\begin{gathered}
\alpha, \quad \beta, \quad-2 \alpha, \quad-2 \beta, \quad \alpha, \quad \beta \\
\alpha, \quad \beta-\alpha, \quad-2 \alpha, \quad 2 \alpha-2 \beta, \quad \alpha, \quad \beta-\alpha .
\end{gathered}
$$

In terms of the elementary parallelograms this transforms the one with vertices $0,2 \alpha$, $2(\alpha+\beta), 2 \beta$ into the one with vertices $0,2 \alpha, 2 \beta, 2(\beta-\alpha)$ and this is obtained by applying $\left(\begin{array}{cc}1 & 0 \\ -1 & 1\end{array}\right)$ and this ends the proof of Theorem $\mathrm{B}$.

5.16 Examples. We can identify in terms of translation surfaces the three examples of 4.14. The first, which has an automorphism of order 4 is obviously the surface defined by three squares itself. The second is the transform of the first under $\left(\begin{array}{cc}-1 & -1 \\ 1 & 0\end{array}\right)$ or equivalently under $\left(\begin{array}{cc}1 & 0 \\ -1 & 1\end{array}\right)$.

For the third example we note that $\left(\begin{array}{cc}0 & -1 \\ 1 & 1\end{array}\right)$ must act as a rotation on the L-type polygon. From this it is not hard to see that the corresponding surface is the one tiled by six equilateral triangles. 


\section{Conclusion and questions}

There are other families to which we can apply the methods developed in Sections 2, 3 and 4. For instance one can apply the transformations of Aline Aigon to the family of surfaces with an order 3 automorphism and proceed as in Section 3. We can also do this for transforms of the family with an order 4 automorphism we have not considered in this paper. Again for all these we will have an action of $\mathrm{PSL}_{2}(\mathbb{Z})$ generated by half twists. The first obvious question is to describe these also as translation surfaces. For real curves with 3 real components and an order 4 automorphism there is an easy interpretation in terms of "Swiss crosses" (see [Mc]), this will be developed in a forthcoming paper.

At the other end we have $\mathrm{SL}_{2}(\mathbb{R})$ orbits of surfaces tiled by squares and one of the natural questions is: is the action of $\mathrm{SL}_{2}(\mathbb{Z})$ also generated by fractional Dehn-twists (and rotations of fundamental domains)?

In the other direction one can also ask the following. If $(S, \omega)$ is a translation surface and $\left(S^{\prime}, \omega^{\prime}\right)$ is the transform of $(S, \omega)$ under $U \in \mathrm{SL}_{2}(\mathbb{R})$ can one express the Fenchel-Nielsen coordinates of $S^{\prime}$ in terms of those of $S$ ?

In fact we can generalize further and consider primitive Teichmüller disks and ask about the hyperbolic counterpart of the actions of $\operatorname{SL}(S, \omega)$ and $\mathrm{SL}_{2}(\mathbb{R})$.

\section{References}

[Ai] A. Aigon-Dupuy, Half-twists and equations in genus 2. Ann. Acad. Sci. Fenn. Math. 29 (2004), 307-328. Zbl 1074.30039 MR 2097235

[Ai et al.] A. Aigon-Dupuy, P. Buser, M. Cibils, A. F. Künzle and F. Steiner, Hyperbolic octagons and Teichmüller space in genus 2. J. Math. Phys. 46 (3) (2005), 033513. Zbl 1067.81041 MR 2125588

[Bu] P. Buser, Geometry and Spectra of Compact Riemann Surfaces. Progr. Math. 106, Birkhäuser, Boston, MA, 1992. Zbl 0770.53001 MR 1183224

[Bu-Si1] P. Buser and R. Silhol, Geodesics, periods and equations of real hyperelliptic curves. Duke Math. J. 108 (2001), 211-250. Zbl 1019.30042 MR 1833391

[Bu-Si2] P. Buser and R. Silhol, Some remarks on the uniformizing function in genus 2. Geom. Dedicata 115 (2005), 121-133. Zbl 02235504 MR 2180044

[Fa-Kr] H. Farkas and I. Kra, Riemann Surfaces. Grad. Texts in Math. 71, Springer-Verlag, Berlin, Heidelberg, New York 1980. Zbl 0475.30001 MR 0583745

[Gu-Ju] E. Gutkin and C. Judge, Affine mappings of translation surfaces: geometry and arithmetic. Duke Math. J. 103 (2000), 191-213. Zbl 0965.30019 MR 1760625

[Hu-Le1] P. Hubert and S. Lelièvre, Prime arithmetic Teichmüller disks in $\mathscr{H}(2)$. Israel $J$. Math. 151 (2006), 281-321. MR 2214127

[Hu-Le2] P. Hubert and S. Lelièvre, Noncongruence subgroups in $\mathscr{H}(2)$. Internat. Math. Res. Notices 2005 (1) (2005), 47-64. Zbl 1069.30074 MR 2130053 
Vol. 82 (2007)

[Mc] C. T. McMullen, Billiards and Teichmüller curves on Hilbert modular surfaces. J. Amer. Math. Soc. 16 (2003), 857-885. Zbl 1030.32012 MR 1992827

[Ok] T. Okai, Effects of a change of pants decomposition on their Fenchel-Nielsen coordinates. Kobe J. Math. 10 (1993), 215-223. Zbl 0822.57009 MR 1270159

[Si1] R. Silhol, Hyperbolic lego and algebraic curves in genus 2 and 3. In Complex manifolds and hyperbolic geometry (Guanajuato, 2001), Contemp. Math. 311, Amer. Math. Soc., Providence, RI, 2002, 313-334. Zbl 1050.30032 MR 1940178

[Si2] R. Silhol, A functional relation for accessory parameters for genus 2 algebraic curves with an order 4 automorphism. J. London Math. Soc. 71 (2005), 133-145. Zbl 1066.30037 MR 2108252

Received April 21, 2005; revised October 19, 2005

Robert Silhol, Université Montpellier II, Département de Mathématiques, UMR CNRS 5149,

Place E. Bataillon, 34095 Montpellier Cedex 5, France

E-mail: rs@math.univ-montp2.fr 